\title{
Modelling rain forest diversity: The role of competition
}

\author{
C.J. Bampfylde ${ }^{\mathrm{a}, \mathrm{b}, *}$, N.D. Brown ${ }^{\mathrm{a}}$, D.J. Gavaghan ${ }^{\mathrm{c}}$, P.K. Maini ${ }^{\mathrm{b}}$ \\ a Department of Plant Sciences, South Parks Road, Oxford OX1 3RB, UK \\ ${ }^{\mathrm{b}}$ Mathematical Institute, 24-29 St. Giles', Oxford OX1 3LB, UK \\ c Oxford University Computing Laboratory, Wolfson Building, Parks Road, Oxford OX1 3QD, UK
}

Received 2 October 2003; received in revised form 28 January 2005; accepted 17 February 2005

Available online 24 March 2005

\begin{abstract}
Rain forests exhibit enormous species diversity, but the mechanisms for establishing and maintaining such diversity are unknown. Models involving both exploitative and pre-emptive competition have been proposed. We examine two of these models mathematically and show that neither can exhibit species diversity. The inclusion of random fruiting events, together with seedling population decay, can result in both models exhibiting long-term coexistence of many species. However, the parameter values required to simulate such behaviour are more realistic for the pre-emptive competition model than for the exploitative competition model. Our analysis has general implications for all tropical rain forests in that it suggests that a competition-colonisation type trade-off is not a sufficient condition for species coexistence.
\end{abstract}

(C) 2005 Elsevier B.V. All rights reserved.

Keywords: Competition; Diversity; Mathematical model; Metapopulations; Rain forests; Seeding events

\section{Introduction}

There has been much debate about the degree to which the composition of species-rich plant communities is influenced more by chance and history than regulated by competition (Hubbell, 1998, 2001). Recruitment limitation (defined as the failure of a plant species to colonise a suitable vacant site) is potentially

\footnotetext{
* Corresponding author. Present address: Department of Mathematical and Statistical Sciences, University of Alberta, Edmonton, Alta., Canada T6G 2G1. Tel.: +1 780492 4756; fax: +1 780492 8373.

E-mail address: cbampfylde@math.ualberta.ca (C.J. Bampfylde).
}

one of the most important processes permitting coexistence of large numbers of ecologically similar species in a single community (Levins and Culver, 1971; Hurtt and Pacala, 1995). However, there are still comparatively few experimental or observational studies in natural terrestrial ecosystems that have demonstrated its importance (the majority of published studies have examined marine or rocky shore community structures). The problem of finding empirical evidence for recruitment limitation is particularly acute in forests where generation times are typically many decades and community dynamics are therefore difficult to assess over a short timescale. Most studies of recruitment in forest ecosystems have been at too small a spatial and tempo- 
ral scale to permit the role of recruitment limitation to be evaluated (Clark et al., 1999). Much of the support for the recruitment limitation hypothesis comes from theoretical, especially mathematical modelling, studies. One model, developed by Tilman (1994) has been widely invoked as demonstrating a putative mechanism for the maintenance of high species richness and has been cited in support of the importance of recruitment limitation in tropical rain forests (Hubbell et al., 1999; Svenning, 1999). Indeed, Tilman (1994) suggests that his model provides an alternative explanation to that of the neutral theory proposed by Hubbell and Foster (1986) and Hubbell (2001) for species richness in tropical forests.

We review Tilman's (1994) model, its formulation and results below. An analysis of the theoretical underpinning of this model shows that it is not appropriate for the simulation of tropical rain forest tree species dynamics. We present an adaptation of Tilman's model for rain forest dynamics in which we make it impossible for an individual tree to invade a space that is already occupied by another individual and we justify why we believe that this more accurately simulates observed processes of gap colonisation. We modify this model to permit a gap in the forest to be colonised by the fastest growing species present in the seedling bank at that time. Finally, we incorporate mast-fruiting events, characteristic of rain forests in Southeast Asia, into our model, and describe the consequences of a progressive decay in species colonisation rates (simulating the decay in populations of species in the seedling bank over time between fruiting events).

\section{Tilman's model of spatially structured competition}

Tilman's (1994) model is based on a well-known metapopulation dynamics equation (Levins, 1969; Levins and Culver, 1971), and is frequently used for modelling ecosystems. Considering a single sessile species living in a habitat composed of distinct sites, where a site can be occupied by at most one adult, the death of an adult opens up a gap for colonists. The dynamics of site occupancy depends on the mortality rate and the colonisation rate of the species. The dynamics of the entire habitat are the sum of the individual processes of death (mortality) and replacement (colonisation) in each site. Similar models have also been applied to marine populations with sessile adult dynamics coupled to motile juvenile dynamics which contribute to a larval pool able to colonise empty sites in the environment (for example, Roughgarden and Iwasa, 1986). The additional complexity of the juvenile stage is not considered here. The important aspects of Tilman's model are captured by the two-species model which we summarise below. For single and multiple species dynamics, we refer the reader to the original text (Tilman, 1994).

\subsection{Competition between two species}

Tilman models interspecific competition by first considering a two-species model in which the competition is for habitat sites. He defines the superior competitor to be the one that always displaces the inferior competitor when they both occur in a site, and the inferior competitor can neither invade into nor displace the superior competitor from a site. In this case, he uses the following equations to model the dynamics:

$\frac{\mathrm{d} p_{1}}{\mathrm{~d} t}=c_{1} p_{1}\left(1-p_{1}\right)-m_{1} p_{1}$,

$\frac{\mathrm{d} p_{2}}{\mathrm{~d} t}=c_{2} p_{2}\left(1-p_{1}-p_{2}\right)-m_{2} p_{2}-c_{1} p_{1} p_{2}$,

where $p_{1}$ denotes the proportion of the superior competitor. Note that the equation for the superior competitor (1) is as if it lived as a species on its own. The inferior competitor can only colonise sites where both it and species 1 are absent (the $\left(1-p_{1}-p_{2}\right)$ term in (2)), however, species 1 can displace species 2 (the $-c_{1} p_{1} p_{2}$ term in (2)). Tilman shows that globally stable coexistence is possible under certain parameter conditions (Tilman, 1994).

\subsection{The applicability of Tilman's model to tropical rain forest communities}

We believe the use of Tilman's model for spatially structured competition to explain rain forest tree species diversity is inappropriate for a number of reasons. Firstly, his assumptions that individuals can displace each other is not realistic for most climax rain forest species, however, relevant this may be elsewhere, for example, in grasslands. We address this assumption 
in Section 3. Secondly, Tilman's model is an equilibrium model. It has been accepted that apparently stable populations are not at equilibrium over some timescales (Hutchinson, 1961). One hypothesis for ecosystem diversity is that it is maintained by recovery from disturbances (Connell, 1978). It follows under this hypothesis, that an equilibrium model is not valid. However, in order to assess whether a model based on this simplification produces the correct type of dynamics, we adapt Tilman's model in Section 3.1. But go on to develop a non-equilibrium or dynamic equilibrium model (sensu Huston, 1979), where we search for a balance between proliferation and death of species between disturbance events (Section 5).

\section{A new model for competition in rain forests}

Tree regeneration in tropical rain forests is typically light limited and most tropical rain forest trees require light gaps in order to grow to maturity (Hartshorn, 1978). Recent analyses of patterns of regeneration in natural tropical rain forest light gaps (Whitmore and Brown, 1996; Delissio et al., 2002) have found that, contrary to widely accepted generalisations, the dominance hierarchy in light gaps is a function of initial seedling size rather than competitive ability for light. Competition for light in gaps is highly asymmetric. The tallest individual at the time of gap creation will benefit from increased levels of irradiance, regardless of species. Those in its shade will not. As a consequence, initial differences in size are often sufficient to overcome any innate differences in competitive ability. When the outcome of competition for limiting resources is determined by initial conditions, it is termed founder control (Keddy and Shipley, 1989). Rees and Bergelson (1997) have shown that asymmetric competition for light increases the likelihood that plants with similar regeneration niches will be founder controlled. The implication of this for rain forest tree dynamics is that once an individual has established in a light gap it is unrealistic to assume that it can be displaced by another individual that is a superior competitor.

It is well known that where big gaps are made in a tropical rain forest, they are often colonised by pioneer tree species (sensu Swaine and Whitmore, 1988). Many shade-tolerant climax tree species are capable of survival and growth beneath pioneer trees (Howlett et al., 1996) and may, over time, replace them in a successional sequence (Kochummen, 1996; Whitmore, 1998). In these circumstances, it may be legitimate to propose that there has been competitive displacement and that Tilman's (1994) model provides an adequate description. Our objective, however, is not to model this type of secondary succession, but to evaluate mechanisms that will permit coexistence of species within the climax guild that are similar in their ecology.

In order to increase the applicability of Tilman's two-species model to rain forest regeneration dynamics, we have adapted it in two important ways. Firstly, we have removed the $-c_{1} p_{1} p_{2}$ term in (2) that permits a species to invade the space occupied by an inferior competitor and displace it. Secondly, we have introduced a component to our model that selects for the species, present at the time of gap formation, that has the highest growth rate. We refer to this model as our "exploitative competition model".

\subsection{Two-species model}

\subsubsection{Model and determination of steady states}

The two competing tree species model lends itself to a complete mathematical study and will help in gaining a deeper understanding of this type of interaction when we incorporate more species.

The two-species system is:

$$
\begin{aligned}
& \frac{\mathrm{d} p_{1}}{\mathrm{~d} t}=c_{1} p_{1} g-m_{1} p_{1}, \\
& \frac{\mathrm{d} p_{2}}{\mathrm{~d} t}=c_{2} p_{2} g\left(1-p_{1}\right)-m_{2} p_{2}, \\
& \frac{\mathrm{d} g}{\mathrm{~d} t}=-c_{1} p_{1} g-c_{2} p_{2} g\left(1-p_{1}\right)+m_{1} p_{1}+m_{2} p_{2},
\end{aligned}
$$

$1=p_{1}+p_{2}+g$

where $p_{1}(t)$ and $p_{2}(t)$ are the proportional abundances of species 1 and 2, respectively, at time $t$, and $g(t)$ is the proportional abundance of available free space (gaps). $c_{i}$ is the colonisation rate and $m_{i}$ is the mortality (local extinction) rate of species $i$. We assume, for the moment, that the $m_{i}$ and $c_{i}$ are positive constants. Adding (3)-(5), we have that $p_{1}+p_{2}+g=$ constant, which 
we take to be 1 (since $p_{1}, p_{2}$ and $g$ are proportions), leading to Eq. (6).

Species 1 is defined to be the most competitive species, that is, the one whose seedlings will grow the fastest to reach the light (in a treefall gap). To derive Eq. (3), propagules are assumed to be dispersed randomly among all sites. The rate of propagule production by the occupied sites of species $1, c_{1} p_{1}$, is multiplied by the proportion of sites that are unoccupied, $g$, to give the rate of production of newly colonised sites (Law of Mass Action). The mortality rate, $m_{1}$, is multiplied by the proportion of sites occupied by species $1, p_{1}$, to give the rate at which the sites become vacant. A site is vacated when the individual occupying it dies.

The introduction of a second species does not affect the dynamics of species 1 , since the new species is an inferior competitor. We assume that the superior competitor always out-competes the inferior competitor if both are present when a gap is formed. We capture this behaviour phenomenologically by assuming that the colonising power of species 2 in the presence of species 1 is $c_{2} p_{2}\left(1-p_{1}\right)$. Multiplying this by the proportion of vacant sites $g$ then gives us the first term on the right-hand side of (4).

Eq. (5) decouples from the system and using (6) we are left with a system of two first-order nonlinear ordinary differential equations:

Table 1

Steady states of (7) and (8) and eigenvalues of the linearised system ( $\hat{\lambda}_{1}$ and $\hat{\lambda}_{2}$ are given in Appendix A)

\begin{tabular}{lllll}
\hline State & $p_{1}^{0}$ & $p_{2}^{0}$ & $\lambda_{1}$ & $\lambda_{2}$ \\
\hline (a) & 0 & 0 & $c_{1}\left(1-\gamma_{1}\right)$ & $c_{2}\left(1-\gamma_{2}\right)$ \\
(b) & 0 & $1-\gamma_{2}$ & $c_{1}\left(\gamma_{2}-\gamma_{1}\right)$ & $c_{2}\left(\gamma_{2}-1\right)$ \\
(c) & $1-\gamma_{1}$ & 0 & $c_{1}\left(\gamma_{1}-1\right)$ & $c_{2}\left(\gamma_{1}^{2}-\gamma_{2}\right)$ \\
(d) & $1-\frac{\gamma_{2}}{\gamma_{1}}$ & $\frac{\gamma_{2}}{\gamma_{1}}-\gamma_{1}$ & $\hat{\lambda}_{1}$ & $\hat{\lambda}_{2}$ \\
\hline
\end{tabular}

species. The state (a) corresponds to both species dying out and no trees being present; (b) corresponds to species 1 being totally dominated by the less competitive species 2, only trees from species 2 are present and there is no coexistence. Similarly in (c), the more competitive species 1 dominates species 2 causing it to die out completely. Finally in (d), both species coexist and both have a non-zero abundance at the steady state. In a rain forest, many species coexist and it is a steady state of type (d) that we are trying to reproduce. The stability and feasibility of these steady states will be investigated below.

\subsubsection{Linear stability analysis, stability and feasibility of steady states}

We now carry out linear analysis of the steady states in Table 1 in the standard way (see, for example, Jordan and Smith, 1988).

The Jacobian matrix, $\boldsymbol{J}_{2}$, of the system is given by:

$$
\boldsymbol{J}_{2}=\left(\begin{array}{cc}
c_{1}\left(1-2 p_{1}-p_{2}-\frac{m_{1}}{c_{1}}\right) & -c_{1} p_{1} \\
-c_{2} p_{2}\left(2-2 p_{1}-p_{2}\right) & c_{2}\left(\left(1-p_{1}\right)\left(1-p_{1}-2 p_{2}\right)-\frac{m_{2}}{c_{2}}\right)
\end{array}\right),
$$

$$
\begin{aligned}
\frac{\mathrm{d} p_{1}}{\mathrm{~d} t} & =c_{1} p_{1}\left(1-p_{1}-p_{2}\right)-m_{1} p_{1}, \\
\frac{\mathrm{d} p_{2}}{\mathrm{~d} t} & =c_{2} p_{2}\left(1-p_{1}-p_{2}\right)\left(1-p_{1}\right)-m_{2} p_{2} .
\end{aligned}
$$

The steady states of the system are found by setting:

$$
\frac{\mathrm{d} p_{1}}{\mathrm{~d} t}=0=\frac{\mathrm{d} p_{2}}{\mathrm{~d} t} .
$$

The four possible steady states are given in Table 1 . For ease of notation, we define $\gamma_{i}=\frac{m_{i}}{c_{i}}$ for $i=1,2$.

The different steady states (or equilibrium points) correspond to different proportional abundances of the evaluated at the steady state $\left(p_{1}^{0}, p_{2}^{0}\right)$. The eigenvalues for each steady state are calculated from $d(\lambda)=\mid \boldsymbol{J}-$ $\lambda \boldsymbol{I} \mid=0$ and shown in Table 1.

The $p_{i}$ represent proportional abundances of rain forest tree species. Therefore, to be a feasible state, both $p_{1}$ and $p_{2}$ must be non-negative. For example, steady state (a) is feasible for all values of $\gamma_{1}$ and $\gamma_{2}$. For steady state (b), to be feasible, we require $1-\gamma_{2}>0$, that is $\gamma_{2}<1$, and similarly for steady state (c), we require $\gamma_{1}<1$. For steady state (d), we need $1-\frac{\gamma_{2}}{\gamma_{1}}>0$ and $\frac{\gamma_{2}}{\gamma_{1}}-\gamma_{1}>0$. Combining the two inequalities gives $0<$ $\gamma_{1}^{2}<\gamma_{2}<\gamma_{1}<1$.

Steady states may be classified by their stability eigenvalues. If there exists at least one eigenvalue with 
real part greater than zero, then there are solutions that are growing exponentially and hence the steady state is linearly unstable. In order for a steady state to be linearly stable, both eigenvalues must have negative real parts.

The results for feasibility and stability are summarised in Fig. 1. From this figure, we see that linear theory predicts that species coexistence cannot occur in this model, i.e. the coexistence steady state is unstable for all feasible parameter values.

The above analysis determines the local behaviour near equilibria. To investigate the global behaviour, we considered the phase plane of the system. A detailed numerical study of the phase plane for the full nonlinear model verifies the prediction that species coexistence cannot occur in this model. Within the parameter regime where coexistence in feasible but unstable, we observe a separatrix in the phase plane dividing the trajectories into the basins of attraction of the two stable single species steady states.

We conclude that the two-species model cannot exhibit the type of behaviour observed in the rain forest. We now use the insights obtained from this model to investigate the extension to the $n$-species model (7)-(8).

\section{2. $n$-Species competition model}

We now investigate an $n$-species competition model to determine whether multiple species $(\geq 3)$ coexistence can be stable.

\subsubsection{Model and determination of steady states}

Extending the model derived in Section 3.1, the system of nonlinear ordinary differential equations be-

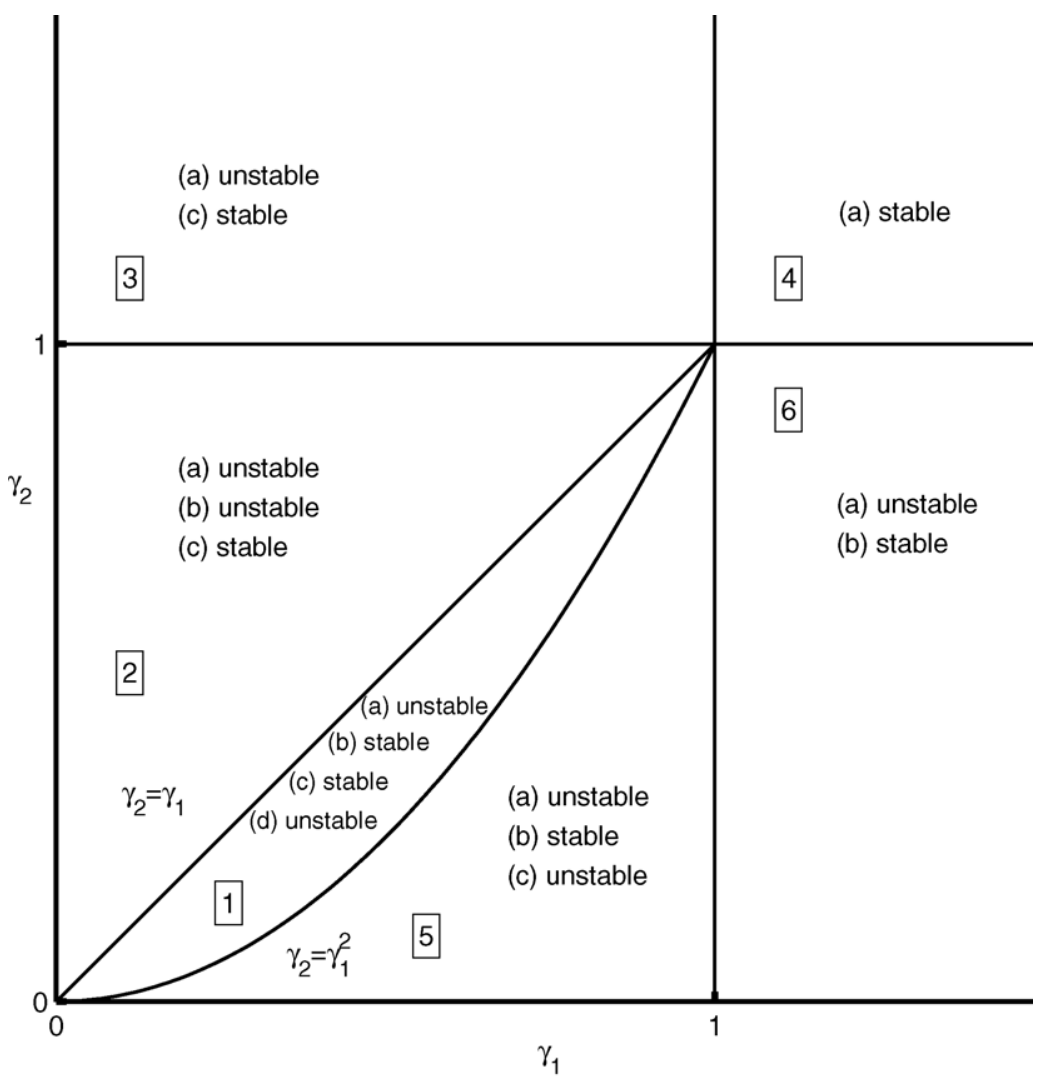

Fig. 1. Stability and feasibility regions. There are six different regions in the $\left(\gamma_{1}, \gamma_{2}\right)$ parameter space. In region $(1), 0<\gamma_{1}, \gamma_{2}<1$, so (a) is unstable, (b) and (c) are feasible and stable (since $\gamma_{2}<\gamma_{1}$ and $\gamma_{2}>\gamma_{1}^{2}$ ), and (d) is feasible but, $\operatorname{Re}\left(\hat{\lambda}_{i}\right)>0$ in this region, thus the steady state is unstable. The other regions are similarly defined. 
comes:

$$
\begin{aligned}
& \frac{\mathrm{d} p_{i}}{\mathrm{~d} t}=c_{i} p_{i} g\left(1-\sum_{j=1}^{i-1} p_{j}\right)-m_{i} p_{i} \\
& \frac{\mathrm{d} g}{\mathrm{~d} t}=-\sum_{i=1}^{n}\left[c_{i} p_{i} g\left(1-\sum_{j=1}^{i-1} p_{j}\right)\right]+\sum_{i=1}^{n} m_{i} p_{i} \\
& 1=g+\sum_{i=1}^{n} p_{i}
\end{aligned}
$$

for $i=1, \ldots, n$.

It is interesting to note that a very similar model has been developed independently, in theoretical immunology, to investigate the mechanisms that maintain size and diversity of pools of $\mathrm{T}$ memory cells. These are cells that enable the immune system to respond more rapidly and with a stronger effect to re-exposure to antigens or pathogens (Yates and Callard, 2001; Callard et al., 2003).

The steady states are calculated in the same way as before by setting the right-hand side of Eq. (10) to zero after eliminating $g$. However, for our purposes, we are interested only in the steady state where all the species have a non-zero abundance, that is all species coexist, because the aim of our model is to predict long-term coexistence of rain forest tree species. In this case, the proportional abundances of the species are calculated to be:

$p_{1}^{0}=1-\frac{\gamma_{2}}{\gamma_{1}}$

$p_{i}^{0}=\frac{\gamma_{i}}{\gamma_{1}}-\frac{\gamma_{i+1}}{\gamma_{1}}, \quad$ for $\quad i=2, \ldots, n-1$,

$p_{n}^{0}=\frac{\gamma_{n}}{\gamma_{1}}-\gamma_{1}$,

where $\gamma_{i}=\frac{m_{i}}{c_{i}}$.

\subsubsection{Stability analysis}

To find the general form of the Jacobian matrix, $\boldsymbol{J}_{n}$, we rewrite the system of Eqs. (10)-(12), replacing $\frac{m_{i}}{c_{i}}$ by $\gamma_{i}$. We obtain the system:

$$
\frac{\mathrm{d} p_{i}}{\mathrm{~d} t}=c_{i} p_{i}\left(1-\sum_{k=1}^{n} p_{k}\right)\left(1-\sum_{k=1}^{i-1} p_{k}\right)-\gamma_{i} c_{i} p_{i} .
$$

The elements of $\boldsymbol{J}_{n}$ are calculated in Appendix B. We find that the system of Eq. (13) for $n>1$ tends to a steady state with at most one species persisting, that is only one of the species is non-zero and all the other species die out (see Appendix B). The species that dominates will be determined by the initial conditions of the system. Hence, we have proved that our model does not mimic the long-term coexistence and diversity observed in the tropical rain forests. We need therefore to consider an alternative mechanism for simulating this species richness. A possible new approach is the inclusion of fruiting events and non-constant colonisation rates. We explore this later, but first we consider a pre-emptive competition model.

\section{Colonisation model}

\subsection{Description of the model}

The idea that communities are assembled according to rules based on the ecological niches of the component species has recently been challenged by the proposal that changes in the abundance of populations and the composition of communities are the consequence of ecological drift rather than competitive interactions (Hubbell, 1998). In order to examine the effect of there being no difference between species in their competitive ability, we have removed the $\left(1-\sum_{j=1}^{i-1} p_{j}\right)$ term from our original model (10). Species differ only in their ability to colonise gaps. The competitive interactions between species are pre-emptive. We have called this our "colonisation model".

The system of equations for our colonisation model then becomes:

$\frac{\mathrm{d} p_{i}}{\mathrm{~d} t}=c_{i} p_{i} g-m_{i} p_{i}$,

$\frac{\mathrm{d} g}{\mathrm{~d} t}=-\sum_{i=1}^{n} c_{i} p_{i} g+\sum_{i=1}^{n} m_{i} p_{i}$,

$1=g+\sum_{i=1}^{n} p_{i}$

Again, $g$ decouples and we obtain:

$$
\frac{\mathrm{d} p_{i}}{\mathrm{~d} t}=c_{i} p_{i}\left(1-\sum_{j=1}^{n} p_{j}\right)-m_{i} p_{i} .
$$


Note that if we assume $c_{i}=c=$ constant, for all $i$ and $m_{i}=m=$ constant, for all $i$ and set $P=\sum p_{i}$, then summing Eq. (17) over $i$, we see that:

$\frac{\mathrm{d} P}{\mathrm{~d} t}=c P(1-P)-m P$,

which is equivalent to Tilman's model for one species and has a stable steady state at $P=1-\frac{m}{c}$. The model for $\mathrm{T}$ memory cells in the immune system also considers this scenario (Yates and Callard, 2001; Callard et al., 2003).

The analysis of stability and feasibility for (17) is much simpler than for the competition model. We spare the reader all of the details and simply summarise the results. There are two cases:

(1) The $\gamma_{i}$ are all different: In this case, if $\gamma_{i}>1$ for all $i$, then the trivial steady state, in which all the species are zero, is the only ecologically meaningful steady state, and it is globally stable. If, however, $\gamma_{i}<1$ for a subset of the $i$ values, then there is a further set of steady states in which every species is zero, except for $p_{i}=1-\gamma_{i}$. In this case, the trivial steady state is no longer stable, and the non-zero steady state that is linearly stable is the one with the smallest $\gamma_{i}$.

(2) A number of the $\gamma_{i}$ are the same: Without loss of generality, assume that $\gamma_{1}=\gamma_{2}=\cdots=\gamma_{k}=\gamma$, and all the other $\gamma_{i}$ are different. Again, if all the $\gamma_{i}$ and $\gamma$ are greater than 1 , then the trivial steady state is the only meaningful one and it is globally stable. If $\gamma<1$, but all other $\gamma_{i}$ are greater than 1 , then the trivial steady state loses stability. There is now a surface of steady states given by the set:

$$
\begin{aligned}
& S=\left\{p_{j}: p_{1}+p_{2}+\cdots+p_{k}=1-\gamma,\right. \\
& p_{i}>0 \text { for } \quad i=1,2, \ldots, k ; \\
& \left.p_{i}=0 \quad \text { for } \quad i=k+1, \ldots, n\right\} .
\end{aligned}
$$

$S$ is neutrally stable. Thus, if we make a perturbation from an equilibrium point in $S$ to another point in $S$, the system will stay at the new point (which is an equilibrium point also). However, if the perturbation moves the equilibrium point off the surface $S$, then the dynamics of the system attracts the solution back to an equilibrium point on the surface $S$. If $\gamma<1$ but one other $\gamma_{i}<1$ for $i>k$, then there

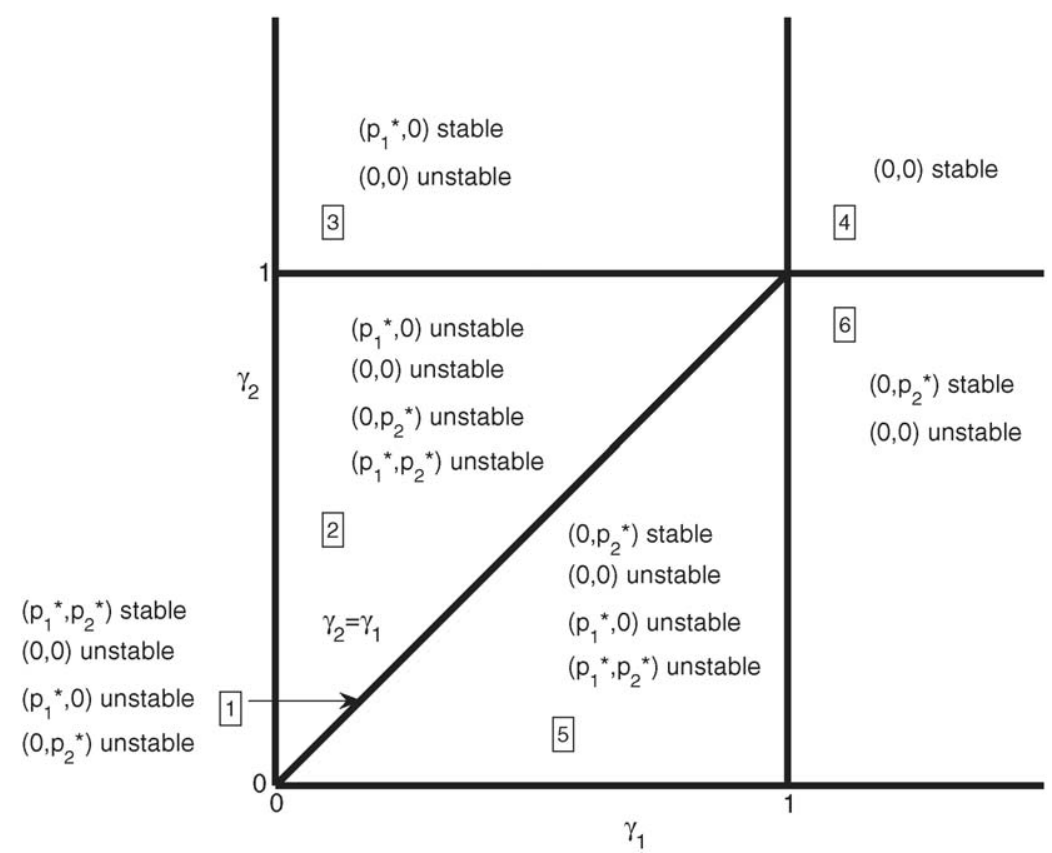

Fig. 2. Stability and feasibility regions. There are six different regions. Region (1) is the line $\gamma_{1}=\gamma_{2}$. The remaining regions are areas marked by the solid lines in the figure. Feasible steady states are given in each region with their stability. The steady states are as follows: $(0,0)$, $\left(p_{1}^{*}, 0\right)=\left(1-\gamma_{1}, 0\right),\left(0, p_{2}^{*}\right)=\left(0,1-\gamma_{2}\right)$, and $\left(p_{1}^{*}, p_{2}^{*}\right)=(1-\gamma, 1-\gamma)$. 
is another steady state in which all the species are zero except for $p_{i}=1-\gamma_{i}$. This new steady state is linearly stable if $\gamma_{i}<\gamma$, otherwise it is unstable.

The results for the stability of the steady states for the specific case of the two-species colonisation model are summarised in Fig. 2. This can be compared to the feasibility and stability regions in Fig. 1.

Numerical simulations of the full nonlinear system support these results (Section 5.4 and Figs. 4-8). We conclude that this model could only possibly give coexistence if some of the $\gamma_{i}$ are equal. However, mathematically speaking, this is the case in which the system is structurally unstable, because any small change in the $\gamma_{i}$ totally changes the behaviour of the system. Such models are of limited validity in an ecological context.

We conclude that our colonisation model, like our (exploitative) competition model, does not adequately simulate the processes that maintain high species diversity.

\section{Fruiting events}

\subsection{Motivation for model}

We have demonstrated that the models described in Sections 3 and 4 cannot support the stable coexistence of species assuming constant values of the prolifera-

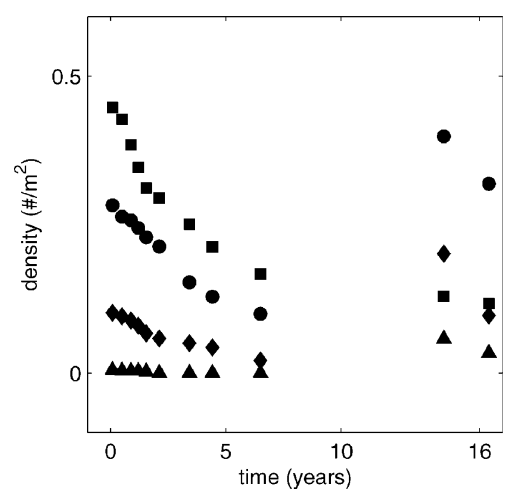

tion rates, $c_{i}$. However, this assumption is unrealistic. Since very few tropical tree species fruit continuously (Lambert and Marshall, 1991) and seedling populations will slowly decay (for an example, see Fig. 3), the proliferation rate for any species is likely to be a function of the time since the last fruiting event. In the New World tropics, the phenology of many trees is highly diverse (Newstrom et al., 1994). This implies that variations between species in propagule abundance and consequently in recruitment may be asynchronous and this will contribute to species coexistence (Chesson and Warner, 1981). Diverse phenology is not an appropriate assumption for Southeast Asian tropical rain forests which are dominated by the Dipterocarpaceae (Whitmore, 1984). An example of the patterns of mast (or gregarious) fruiting (Burgess, 1972) at supra-annual intervals can be seen in Curran et al. (1999, Fig. 1A). The timings of the fruiting events is observed to be approximately periodic with a random component (Yasuda et al., 1999), triggered by the El Niño Southern Oscillation (ENSO) (Sakai et al., 1999). ENSO is not strictly periodic (periodogram analysis; D. Bebber, personal communication) although there is a strong ENSO every 4-7 years (Webster and Palmer, 1997; McPhaden, 1999a,b). In these years, seed and seedling survival are relatively high and mixed species carpets of seedlings are formed often at densities of over one million per hectare. This unique feature of Southeast Asian tropical rain forest makes it problem-

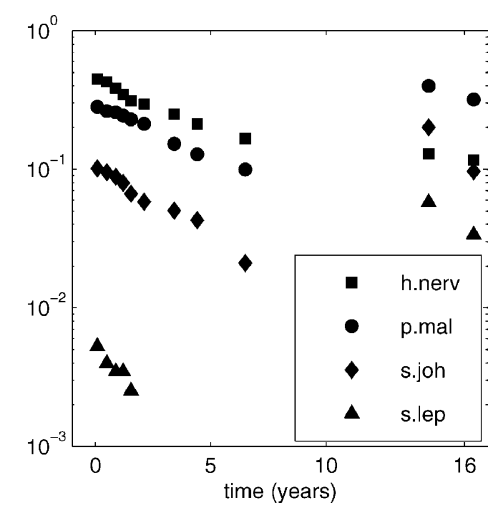

Fig. 3. Seedling bank density decaying over time since a fruiting event. Data collected in Danum Valley, Sabah, Malaysia from 1987 to 2003. Mast-fruiting events were recorded in 1987, 1991 and 1998 (Curran et al., 1999). Four species of Dipterocarpaceae are shown in this example: Hopea nervosa (h.nerv), Parashorea malaanonan (p.mal), Shorea johorensis (s.joh), and S. leprosula (s.lep), with average densities across seven sample plots of differing sizes and light climates. The left-hand panel shows the density on a linear scale, the right-hand panel shows the seedling density on a logarithmic scale. This highlights the exponential decay of seedling density. 
atic to invoke explanations of coexistence for dipterocarps, which depend on asynchronous recruitment (see, for example, the models proposed by Kubo and Iwasa (1996)).

Levins (1979) has previously shown that in a system where global coexistence is not stable and only the trivial steady state is stable, incorporating some environmental variance may lead to global stability of a (coexistence) state. The inclusion of environmental variance is shown to have a similar effect to the inclusion of another resource dimension to the niche space, thus providing opportunities for more species to coexist.

Numerical experiments have also been used to assess the effects of non-equilibrium conditions on competing species. Periodic population reductions have been used as an example of temporal heterogeneity (Huston, 1979). The length of the period of these reductions is adjusted to find a dynamic equilibrium between competitors. Doyle (1981) found that in order to reproduce Puerto Rican montane rain forest dynamics correctly, the disturbance effects of hurricanes needed to be included in his model. Doyle specified the expected reduction in species populations occurring in a given year.

The effects of a random frequency of disturbance events have not been investigated. Our fruiting events (population inputs) are randomly spaced in time. We aim to combine the above elements: randomness, aperiodic disturbance events and variability (with time dependent parameters).

In this section, we therefore introduce random fruiting events and exponential seedling population decay into both our competition and colonisation models, and again investigate if these models will exhibit the longterm coexistence and diversity observed in reality. As it is observed in the field that the seedling bank of each species decays over time between fruiting events, we have changed the colonisation rates in the model (constant until this point) to decay over time at a different rate for each species. We have selected a range of decay rates that are consistent with field observations of the persistence of dipterocarp seedlings (Fox, 1976; Still, 1996; Whitmore and Brown, 1996) and assigned these so that there is an inverse relationship between competitive ability and the rate of population decay. This assumes that there is a trade-off between physiological and morphological traits that enhance seedling persis- tence in the forest understorey and those that enable fast growth in gaps. There is ample experimental evidence that this type of trade-off exists (Bazzaz and Pickett, 1980; Press et al., 1996). These more complex models are analytically intractable, and we therefore resort to extensive numerical simulation to investigate their behaviour within the parameter space.

\subsection{Description of model}

\subsubsection{Colonisation rates}

The number of seedlings, of any particular species, decays with time over the course of a fruiting cycle. Data collected indicates that this decay is exponential (Brown et al., 1999 and Fig. 3) and thus we take the colonisation rates to be of the following form:

$c_{i}=\alpha_{i} \mathrm{e}^{-\beta_{i} t}$,

where $\beta_{i}$ is the decay rate of the seedling population of species $i$ per year. The new meaning of $c_{i}$ is that it is the absolute colonisation power of species $i$, that is the ability of a seedling of species $i$ to establish itself in a site given that the species has been recruited to a site. $\alpha_{i}$ is the initial value of the colonisation power for species $i$ at the start of every fruiting cycle.

\subsubsection{Model equations for the exploitative} competition hypothesis with fruiting events

Our new model for $n$ species then becomes:

$$
\begin{aligned}
\frac{\mathrm{d} p_{1}}{\mathrm{~d} t} & =c_{1}(t) p_{1} g-m_{1} p_{1}, \\
\frac{\mathrm{d} p_{i}}{\mathrm{~d} t} & =c_{i}(t) p_{i} g\left(1-\sum_{j=1}^{i-1} p_{j}\right)-m_{i} p_{i},
\end{aligned}
$$

$\frac{\mathrm{d} g}{\mathrm{~d} t}=-\sum_{i=1}^{n}\left[c_{i}(t) p_{i} g\left(1-\sum_{j=1}^{i-1} p_{j}\right)\right]+\sum_{i=1}^{n} m_{i} p_{i}$,

$\frac{\mathrm{d} c_{i}}{\mathrm{~d} t}=-\beta_{i} c_{i}(t)+\alpha_{i} \delta(t-I)$,

$1=g+\sum_{j=1}^{n} p_{j}$ 
for $i=2, \ldots, n$, where $\delta(t-I)$ is a Dirac delta function, such that:

$\delta(t-I)= \begin{cases}0, & \text { if } t \neq I, \\ \infty, & \text { if } t=I .\end{cases}$

A property of the Dirac delta function is when $\delta(t-I)$ is integrated the term is equal to 1 when $t=I$. $I$ is an indicator function:

$I=I\left(t \in U_{1}, U_{2}, U_{3}, \ldots\right)$,

where $U_{k}$ are a sequence of random variables such that $U_{1}$ is distributed uniformly in $[3,11]$ and $U_{k+1}=U_{k}+$ $u$, where $u$ is also distributed uniformly in $[3,11]$.

On eliminating $g$, Eqs. (20)-(24) become:

$$
\begin{aligned}
& \frac{\mathrm{d} p_{1}}{\mathrm{~d} t}=c_{1}(t) p_{1}\left(1-\sum_{j=1}^{n} p_{j}\right)-m_{1} p_{1}, \\
& \frac{\mathrm{d} p_{i}}{\mathrm{~d} t}=c_{i}(t) p_{i}\left(1-\sum_{j=1}^{n} p_{j}\right)\left(1-\sum_{j=1}^{i-1} p_{j}\right)-m_{i} p_{i},
\end{aligned}
$$

$\frac{\mathrm{d} c_{i}}{\mathrm{~d} t}=-\beta_{i} c_{i}(t)+\alpha_{i} \delta(t-I)$,

for $i=2, \ldots, n$. The inclusion of $c(t)$ into the model equations is equivalent to adding $\alpha_{i}$ individuals into each species $i$ population at randomly distributed time points (i.e. a pulse originating from the $\delta(t-I)$ component in Eq. (29)). These represent the fruiting events.

\subsubsection{Model equations for the colonisation}

hypothesis with fruiting events

Extending the pre-emptive competition model (Eqs. (14)-(16)), we include fruiting events in the form of exponential colonisation rates. The model for $n$ species becomes:

$$
\begin{aligned}
& \frac{\mathrm{d} p_{i}}{\mathrm{~d} t}=c_{i}(t) p_{i} g-m_{i} p_{i}, \\
& \frac{\mathrm{d} g}{\mathrm{~d} t}=-\sum_{i=1}^{n}\left[c_{i}(t) p_{i} g\right]+\sum_{i=1}^{n} m_{i} p_{i}, \\
& \frac{\mathrm{d} c_{i}}{\mathrm{~d} t}=-\beta_{i} c_{i}(t)+\alpha_{i} \delta(t-I),
\end{aligned}
$$

$1=g+\sum_{i=1}^{n} p_{i}$

for $i=1, \ldots, n$, where $I$ is given as above for the exploitative competition model. Or, eliminating $g$,

$\frac{\mathrm{d} p_{i}}{\mathrm{~d} t}=c_{i}(t) p_{i}\left(1-\sum_{j=1}^{n} p_{j}\right)-m_{i} p_{i}$,

$\frac{\mathrm{d} c_{i}}{\mathrm{~d} t}=-\beta_{i} c_{i}(t)+\alpha_{i} \delta(t-I)$.

\subsection{Problems with determination of parameter values}

Unfortunately, there are very few experimental datasets available to indicate the appropriate values for our colonisation parameters $\left(c_{i}, \alpha_{i}\right.$, and $\left.\beta_{i}\right)$ and to a lesser extent, the mortality rates $\left(m_{i}\right)$. In deriving parameter values for his model, Tilman (personal communication) first explored his model (Eqs. (1) and (2)) theoretically to determine the plausibility of a colonisation-competition mechanism to explain coexistence. From this he determined the necessary relationships among parameters for coexistence. He then set the colonisation ability of the dominant species and explored the general phenomenon, using data on competitive abilities and seed production rates to estimate the parameters. His parameter values led to a predicted successional dynamics and coexistence that seemed to mimic qualitatively the patterns he had observed in his grasslands.

More recently, further study and experiments have been devoted to determining competition coefficients (parameters describing the competitive effect one species has on another). In our model, this is incorporated into the colonisation rates. These parameters are difficult to measure due to the fact that plants need to be grown in mixtures and separately to determine their values (Freckleton and Watkinson, 2000, 2001; Weigelt and Jolliffe, 2003). We do not have data regarding controlled experiments with our study species growing in mixtures or in monoculture, but we do have some data on seedling production and longevity of species in the seedling bank following a fruiting event. We use this to estimate colonisation and decay rates. Data analysis and parameter estimation is found elsewhere (Bampfylde, 1999). Following Tilman, we have 
attempted to find the appropriate range of parameters, based on the experimental values that are available (Whitmore and Brown, 1996; Brown et al., 1999). By comparing the magnitude of proliferation $\left(c_{i}, \alpha_{i}, \beta_{i}\right)$ and the decay $\left(m_{i}\right)$ parameters, we were able to determine the likely range of each parameter based on the experimental data available (more details of this calculation are given in Appendix D). We then explored this space numerically to determine the effects of each of the parameters, and more particularly, whether any set of parameters could give long-term coexistence for large numbers of species in the presence of random fruiting events. The results are presented in Section 5.4, for 2, 6 and 180 species.

\subsection{Numerical experiments}

\subsubsection{Competition model}

Our system, Eqs. (27)-(29), is solved over the length of a fruiting interval. The fruiting interval is a random amount of time after which the seedling bank is replenished and the $c_{i}(i=1, \ldots, n)$ are reset to their initial values, taking the current values of $\left(p_{1}, \ldots, p_{n}\right)$ as the new initial conditions in order to solve the system over the next interval.

The time between fruiting intervals is chosen uniformly on the interval $[3,11]$. This is equivalent to events occurring on a periodic frequency with a random time component. This range of values agrees with experimental data (Burgess, 1972), however, there is not enough data available to determine the distribution of masting events hence a uniform distribution is taken. Further experiments using a normal distribution yielded similar results.

The system of nonlinear equations is solved using the MatLaB (MathWorks and Inc., 2002) ode45 (an explicit, one-step Runge-Kutta medium order (4-5) ODE solver) from the odetoolbox (Shampine and Reichelt, 1997). The system of equations for the nonfruiting events model is solved using MATLAB ode15s
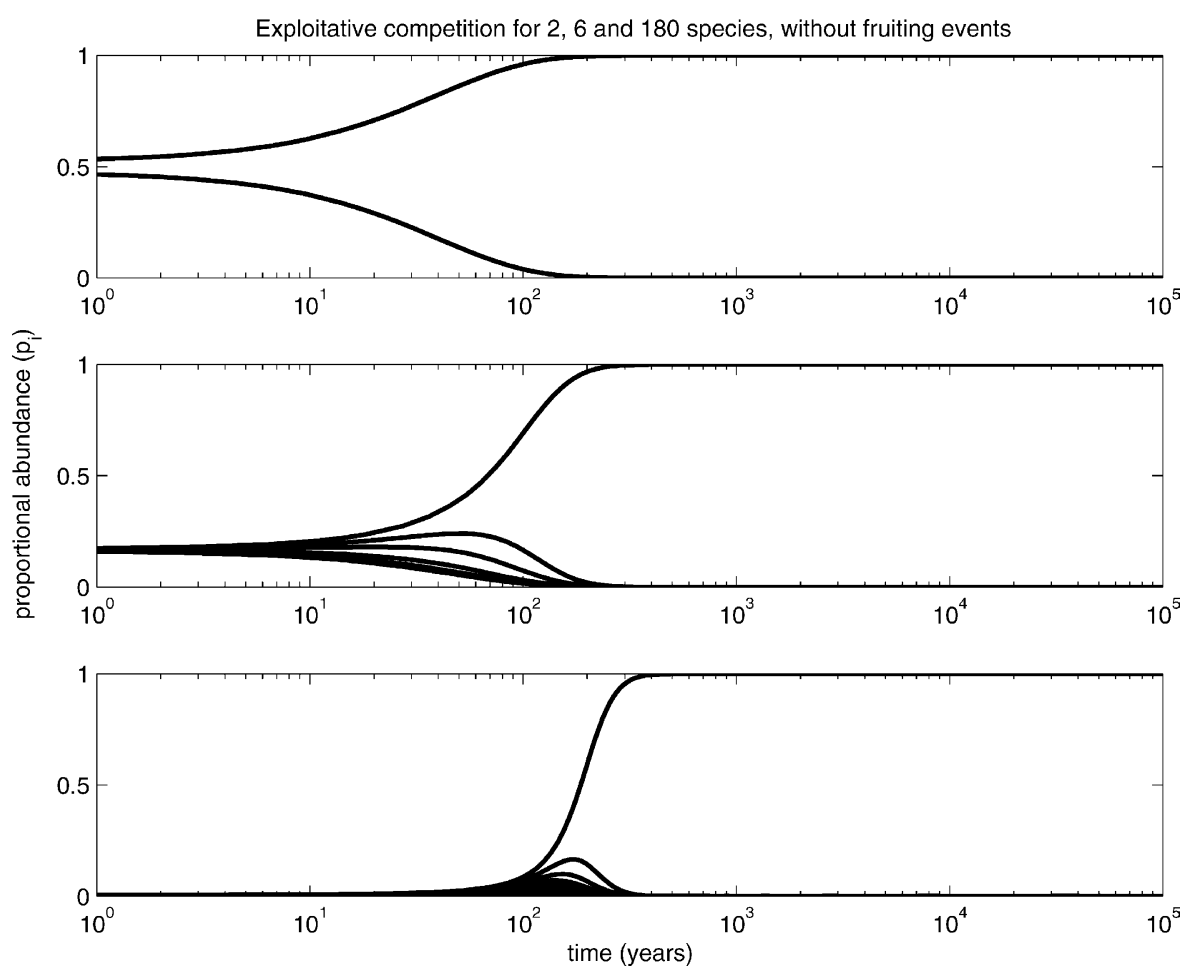

Fig. 4. Dynamics of competition between species, for our exploitative competition model (10)-(12) without fruiting events. Parameter values are given in Table E.1 ( $m_{i}$ and $\overline{c_{i}}$, Appendix E). In the bottom panel, the superior competitor (increasing line) rapidly reaches fixation. In each case, we observe that the superior competitor excludes less competitive species, while the timescale to fixation increases with the number of species included in the simulation. Note the logarithmic scale on the horizontal (time) axis. 
(an implicit multi-step numerical ODE solver of varying order (1-5)). ode15 $\mathrm{s}$ is used as an ODE solver for stiff problems, i.e. when variables are changing values on very different timescales (Byrne and Hindmarsh, 1987; Trefethen, 1996). This is required for the nonfruiting events simulations.

Fig. 4 shows the effect of constant colonisation and no fruiting events (for 2, 6 and 180 species). This simulates Eqs. (10)-(12), with constant average values for the $c_{i}$, given by $\bar{c}_{i}$ in the tables of parameter values (see Appendix E), over the length of a fruiting interval. The calculation of the $\bar{c}_{i}$ is discussed in Appendix F. The reader should note that the equilibrial abundance of the most dominant species $(i)$ is not 1 , but $1-\gamma_{i}$, but because of the scale and parameter values used, it is not possible to distinguish the difference.

Fig. 5 shows numerical solutions of the exploitative competition model with fruiting events, simulating
Eqs. (20)-(24) with the parameter values given in Table E.1 (Appendix E) and the assumption that colonisation rates decrease with time.

The simulation for the two-species model appears to exhibit coexistence. Let us now consider the twospecies case separately. Fig. 6 shows three parameter regimes with different outcomes: coexistence; superior competitor dominates; and inferior competitor dominates. The corresponding phase plane for each regime is also given. In Fig. 6B, trajectories move towards both stable fixed points alternately, however, the random perturbations introduced into the system are sufficient to halt competitive exclusion. Comparing Fig. 6B to numerical investigations of the phase plane, the trajectories 'bounce' from either side of the separatrix.

While it has been possible to obtain long-term coexistence (in particular, in the two-species case), we are
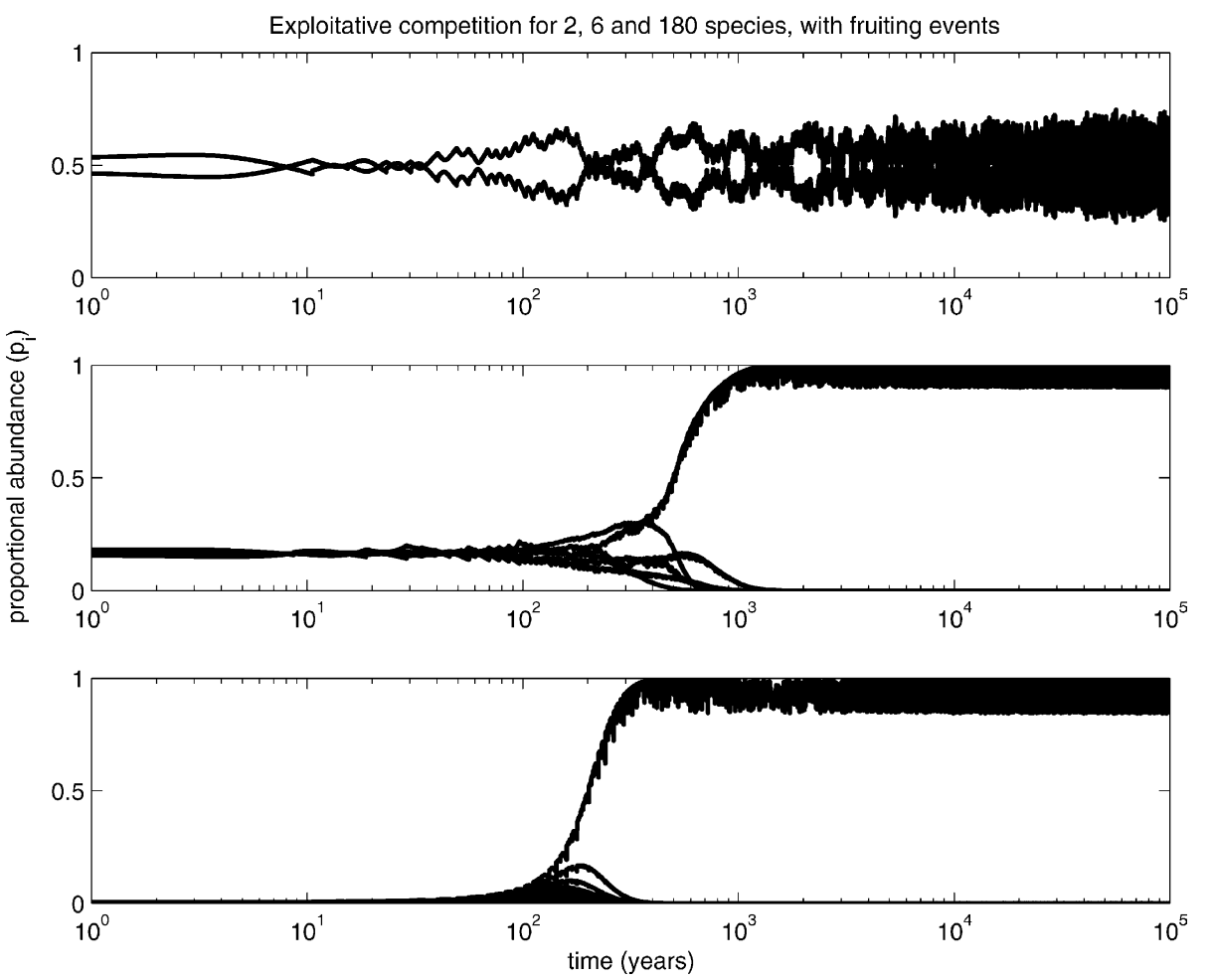

Fig. 5. Dynamics of competition between species, for our exploitative competition model (20)-(24) with fruiting events. Parameter values are given in Table E.1 $\left(m_{i}, \alpha_{i}\right.$ and $\beta_{i}$, Appendix E). In the bottom panel, the superior competitor (increasing line) rapidly reaches fixation. In the two-species case, manipulation of the parameter values enables us to find a parameter regime where both species coexist. In the 6-species case, 2 species persist after 1000 years, while in the 180-species case, with realistic parameter values, 1 species rapidly dominates the rest. In both the 6-and 180 -species cases, after $10^{4}$ years only 1 species persists. Note the logarithmic scale on the horizontal (time) axis. 


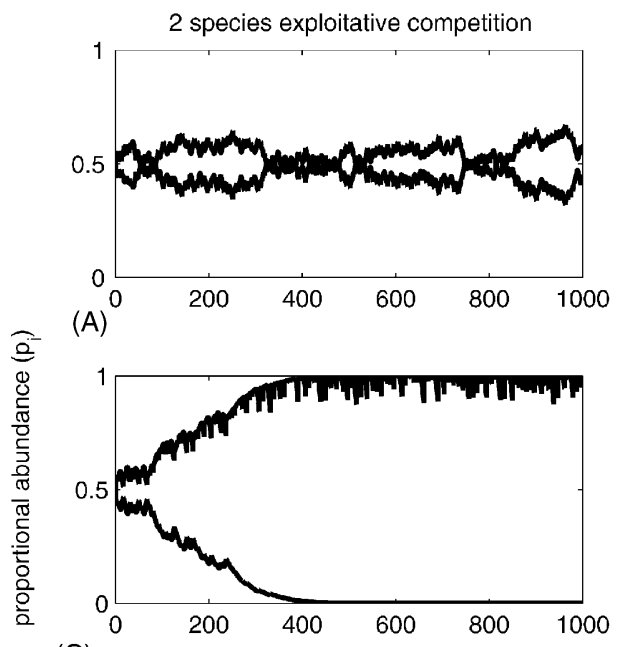

(B)

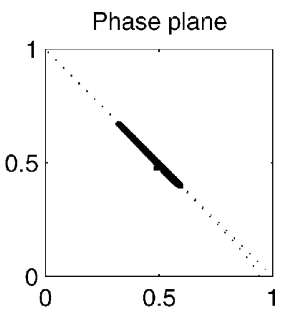

(C)
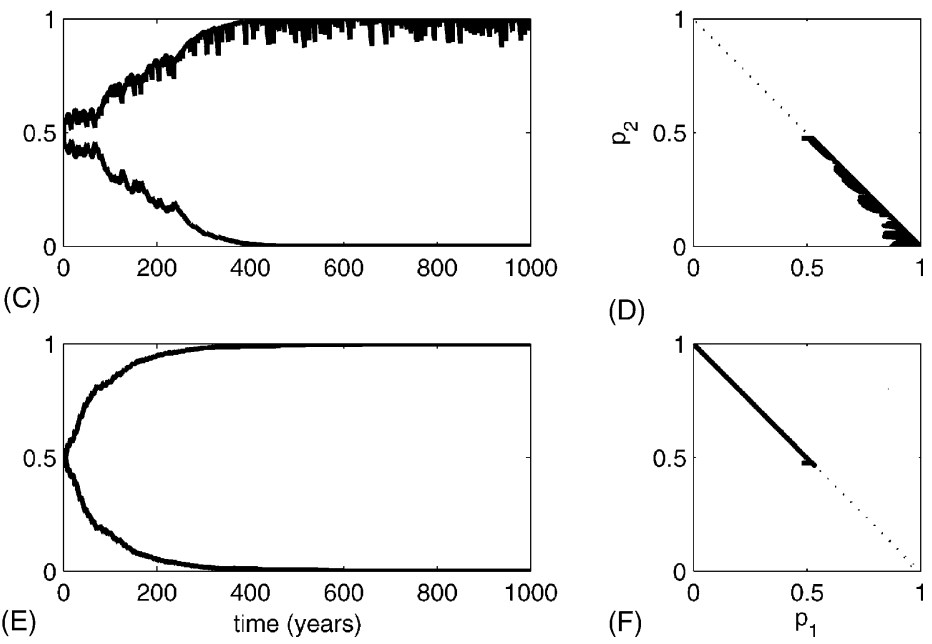

(D)

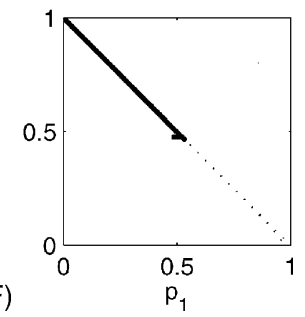

Fig. 6. Dynamics of competition between two species, for the exploitative competition model (20)-(24) with fruiting events. Initial conditions: $\left(p_{1}, p_{2}\right)=(0.475,0.475)$ and $m=0.0255$. ( $\mathrm{A}$ and $\left.\mathrm{B}\right) \alpha_{1}=500, \alpha_{2}=9, \beta_{1}=1.6, \beta_{2}=0.05$, coexistence. $(\mathrm{C}$ and $\mathrm{D}) \alpha_{1}=550, \alpha_{2}=9, \beta_{1}=$ $1.2, \beta_{2}=0.05$, species 1 fixation. (E and F) $\alpha_{1}=350, \alpha_{2}=35, \beta_{1}=2, \beta_{2}=0.05$, species 2 fixation. Panels $\mathrm{A}, \mathrm{C}$ and $\mathrm{E}$ show dynamics of $p_{1}$ and $p_{2}$ as a function of time. Panels B, D and F show the phase plane trajectories (solid lines) with the nullclines of the constant system of equations (dotted lines).

forced to use very unrealistic parameter values (especially for the colonisation decay rates, $\beta_{i}$ ), compared to experimentally collected data (Whitmore and Brown, 1996). Extensive numerical investigation of the parameter space has not yielded a parameter set that gives long-term coexistence and the correct amount of free space for 180 species. If we use realistic parameter values, one species quickly excludes the rest and coexistence is not observed numerically (Fig. 5, bottom panel).

Climax rain forest trees are ecologically very similar and we would expect them to have broadly similar mortality and colonisation rates (parameters $c_{i}, \alpha_{i}, \beta_{i}$ and $m_{i}$ in our models). However, our model required a wide range of parameter values to simulate coexistence. The range is too great to be a likely description of rain forest species.

\subsubsection{Colonisation model}

Fig. 7 shows the effect of constant colonisation and the absence of fruiting events on our colonisation model. The $c_{i}$ used are average values over an average fruiting interval, given by $\bar{c}_{i}$ as shown in Table E.2 (Appendix E). Once again this does not simulate the correct dynamics and so we include fruiting events and exponentially decaying colonisation powers. The results are shown in Fig. 8. Our system, Eqs. (34) and (35), is solved over the length of a fruiting interval. The system of nonlinear equations is solved using MATLAB ode45. Fig. 8 shows the results with the parameter values given in Table E.2.

Let us now consider the two-species case separately as we did for the exploitative competition model in Fig. 6. Fig. 9 shows three parameter regimes with different outcomes: coexistence; superior competitor dominates; 

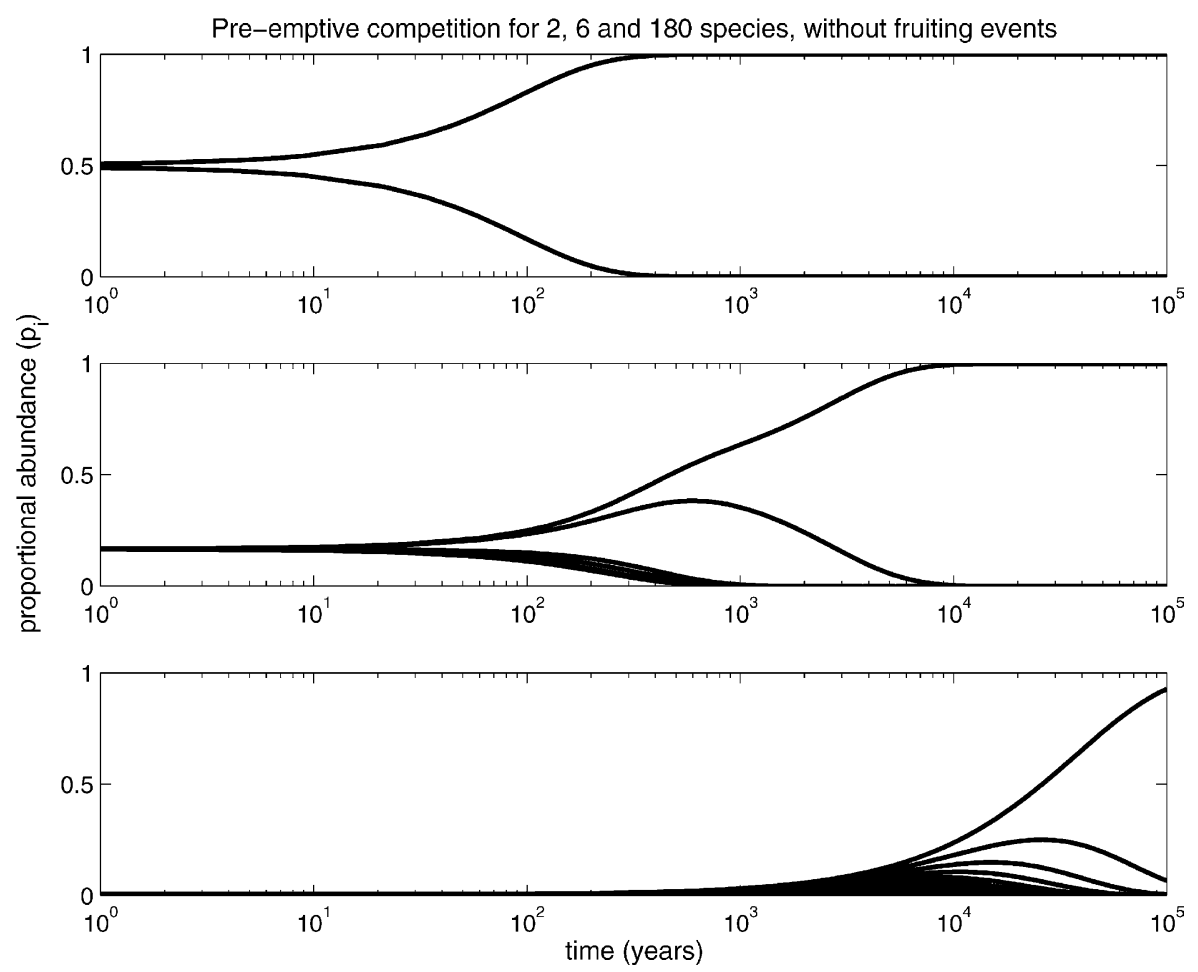

Fig. 7. Dynamics of competition between species, for our colonisation model (17) without fruiting events. The parameter values are given in Table E.2 ( $m_{i}$ and $\overline{c_{i}}$, Appendix E). In the bottom panel, the superior competitor (increasing line) reaches fixation. In each case, we see that the superior competitor excludes less competitive species, while the timescale to fixation increases with the number of species included in the simulation, and these timescales are longer than the corresponding timescales to extinction in Fig. 4. Note the logarithmic scale on the horizontal (time) axis.

and inferior competitor dominates. The corresponding phase plane for each regime is also given. In Fig. 9B, trajectories move towards both stable fixed points alternately, however, the random perturbations introduced into the system are sufficient to halt competitive exclusion.

It is clear from the comparison of Fig. 5 with Fig. 8 that there is a balance between proliferation and death of each species: a dynamic equilibrium (sensu Huston, 1979) for species within our colonisation model but not within our competition model. In addition to this, the parameter values required for coexistence in the simulations for our colonisation model are much more realistic than for our competition model simulations, and are compatible with experimental data (Whitmore and Brown, 1996). We are able to use very similar parameter values for each species, such as might be expected for climax rain forest tree species, and can simulate coexistence over large timescales.

\subsection{Comparison between two-species exploitative and pre-emptive competition models with fruiting events}

The numerical results shown above in Figs. 5 and 8 only include single parameter sets. Next, we investigate the parameter space $\left(\gamma_{1}, \gamma_{2}\right)$, which is shown with regions of stability and feasibility for the exploitative competition model without fruiting events in Fig. 1. Now that the colonisation rates are a function of time, $c_{i}(t)=\alpha_{i} \exp \left(-\beta_{i} t\right)$, there are four parameters associated with the colonisation rates for two species in addition to the mortality rate, $m$. We fix the $\beta_{i}$ and $m$ to reduce the parameter space we need to investigate, this also may allow us a better comparison with Fig. 1 which is in $\left(\gamma_{1}, \gamma_{2}\right)$ space. The results of one set of parameters are shown in Fig. 10. We see which species dominates after 1000 years of the simulation, or whether coexistence is maintained. The relative proportions of each 

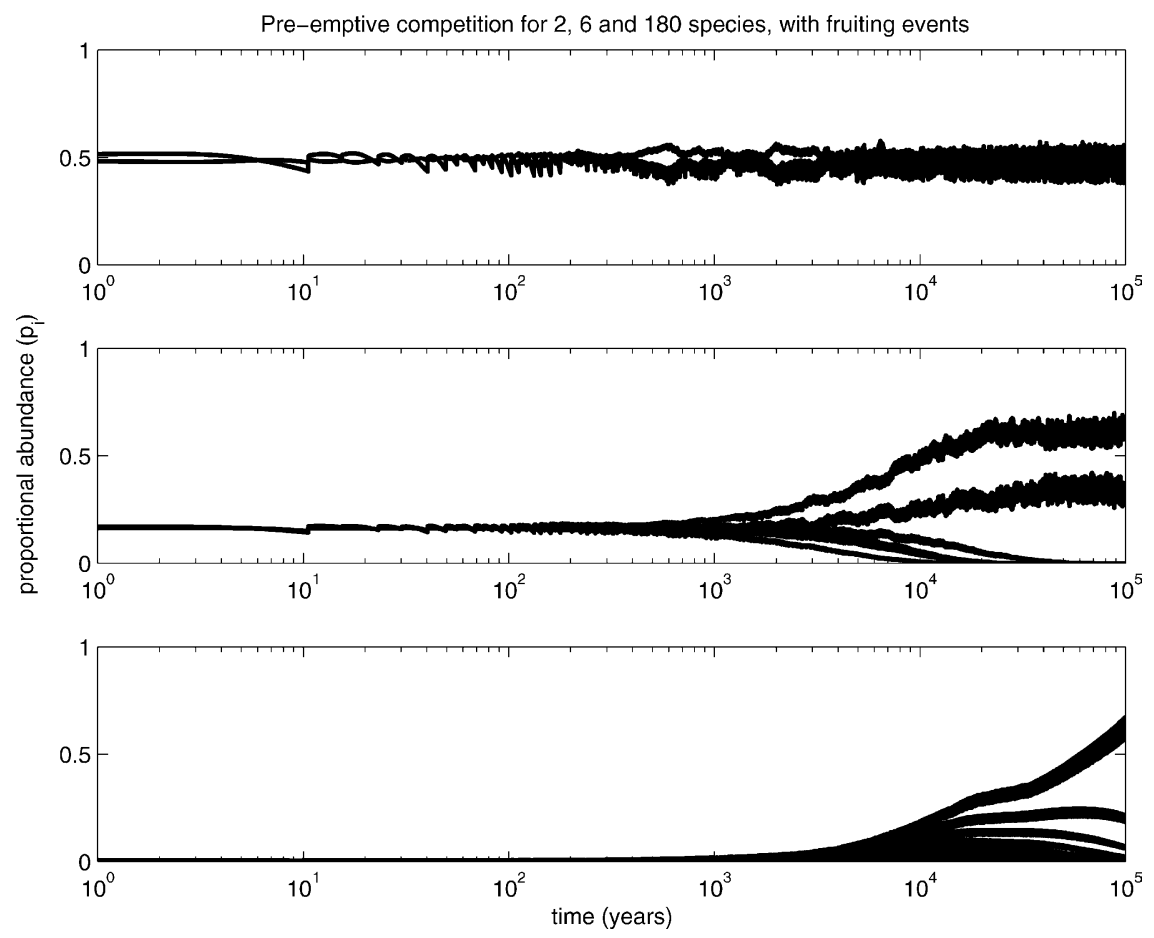

Fig. 8. Dynamics of competition between species, for our colonisation model (30)-(33). The parameter values are given in Table E.2 $\left(m_{i}, \alpha_{i}\right.$ and $\beta_{i}$, Appendix E). In all cases (2, 6 and 180 species), there is a balance between proliferation and death which enables all species to persist over 1000 years, while using realistic parameter values. After $10^{4}$ years, 5 species persist in the 6 -species model and 26 species persist in the 180 -species model. However, after $10^{5}$ years, 2 species persist in the 6-species case, with 6 species persisting in the 180 -species case. Note the logarithmic scale on the horizontal (time) axis.

species in each area of the parameter space are shown (black $=1$, white $=0$ ), along with regions in the parameter space that lead to coexistence. Unfortunately, the parameter region for unstable coexistence (Fig. 1, region 1) in the exploitative competition model does not neatly correspond to that observed when fruiting events are incorporated into the model. Additionally, the area of coexistence in the parameter space in the pre-emptive model does not lie along the line of neutral stability of the coexistence in the deterministic model. This could be due to the fact that the average value of the colonisation rates, $c_{i}$, are not exactly the correct value to use in the comparison with the deterministic models. But what is apparent is that the region of coexistence is greater for pre-emptive over exploitative competition.

Obviously, we have only presented results from a limited region of the entire parameter space $\left(\alpha_{1}, \alpha_{2}, \beta_{1}, \beta_{2}, m_{1}, m_{2}\right)$, but these results are indicative of the general results. That is coexistence is possible for a wider range of parameters in the pre-emptive model as compared to the exploitative competition model.

\subsection{Timescale for decay}

Comparing Fig. 4 with Fig. 7, we observe that the timescale for decay in the exploitative competition model is much less than that for the pre-emptive competition model. In order to quantify the length of the phase of species decline within both of the above models (Eqs. (13) and (17)), we approximate the decay as being exponential and calculate the half-life timescale from the eigenvalues for each system. Details of the calculation are given in Appendix C. We calculate the approximate time taken for all non-dominant species to decay to $1 \%$ of their initial proportion and (in the case of 6 and 180 species) the time for $50 \%$ of all species to decay to $1 \%$ of their initial values. Comparison between numer- 

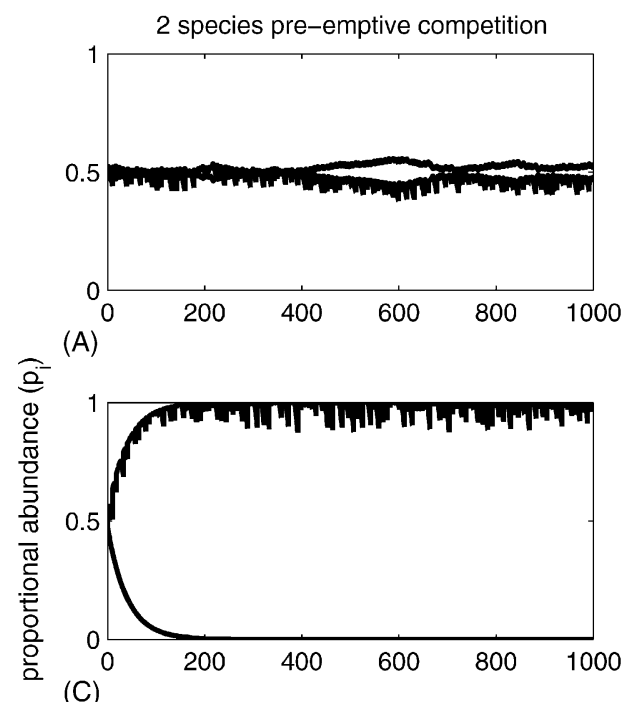

(B)
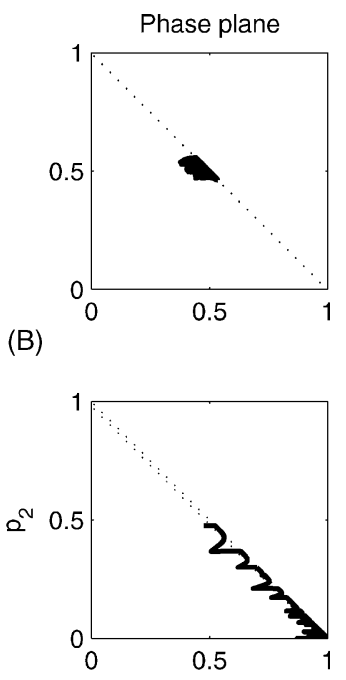

(D)
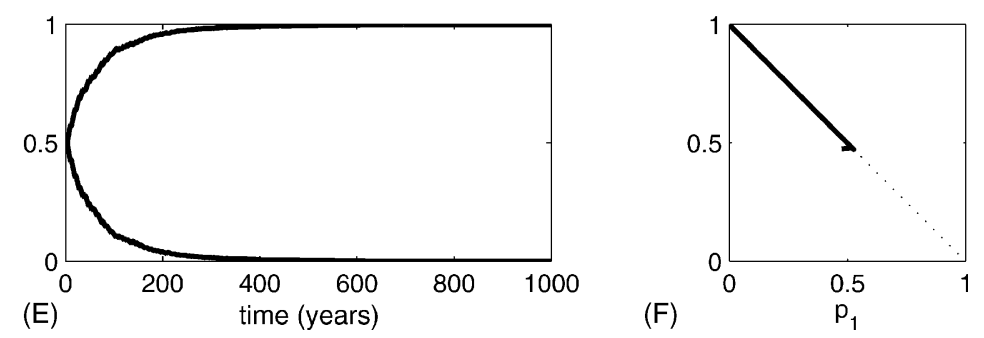

Fig. 9. Dynamics of competition between two species, for the pre-emptive competition model (30)-(33) with fruiting events. Initial conditions: $\left(p_{1}, p_{2}\right)=(0.475,0.475)$ and $m=0.0255$. (A and B) $\alpha_{1}=120, \alpha_{2}=25, \beta_{1}=1.2, \beta_{2}=0.5$. ( $\mathrm{C}$ and D) $\alpha_{1}=550, \alpha_{2}=9, \beta_{1}=1.2, \beta_{2}=$ 1.0. (E and F) $\alpha_{1}=350, \alpha_{2}=35, \beta_{1}=2, \beta_{2}=0.05$. Panels $\mathrm{A}, \mathrm{C}$ and $\mathrm{E}$ show dynamics of $p_{1}$ and $p_{2}$ as a function of time. Panels $\mathrm{B}, \mathrm{D}$ and $\mathrm{F}$ show the phase plane trajectories (solid lines) with the nullclines of the constant system of equations (dotted lines). The nullclines are given by $p_{1}=0, p_{2}=0,1-p_{1}-p_{2}=\gamma_{1}$ and $1-p_{1}-p_{2}=\gamma_{2}$.

ical simulations and our calculated timescales are in close agreement. For our competition model with two species, our calculated time to decay is 210 years and for six species it is 250 years. For 180 species dynamics, the model predicts 350 years for $50 \%$ of the species to decay, and 400 years for one to dominate. These values correspond well to values observed in Fig. 4. The calculated timescale for dominance for our colonisation model is 400 years for 2 species, $1.1 \times 10^{4}$ years for 6 species and $3.7 \times 10^{5}$ years for 180 species. However, the timescale for half of the species to decay is 1000 years for 6 species and 2200 years for 180 species (Fig. 7). Simulated timescales from our numerical experiments are close to these values, however, they are not exact due to the assumption that all species decay exponentially. There are more complex nonlinear com- petitive interactions between the species which may affect the timescale for dominance. This strengthens the case that the pre-emptive model is a more realistic description of competition. This form of competitive interaction slows down the rate of extinction, when coupled with temporal variability (fruiting events) they are sufficient to allow long-term persistence of species in an unstable equilibrium.

\subsubsection{Variability of random results}

The results presented in the graphs with fruiting events (Figs. 5 and 8) are subject to some variation due to the fact that the interval between events is randomly determined at each step. In the case of the exploitative competition model, different competitors come to dominate on different occasions largely due to chance. In 


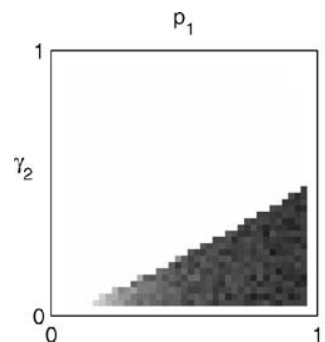

(A)

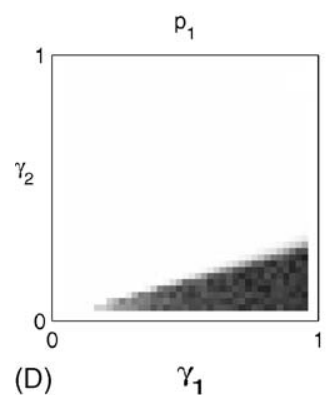

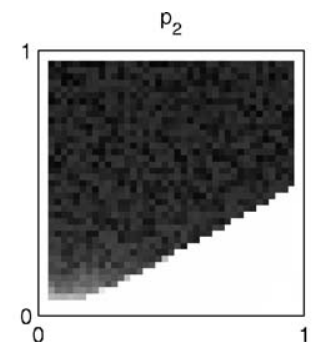

(B)

$\gamma_{1}$

$p_{2}$

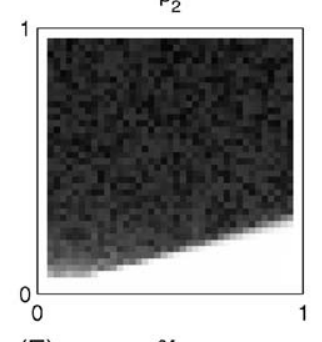

(E)

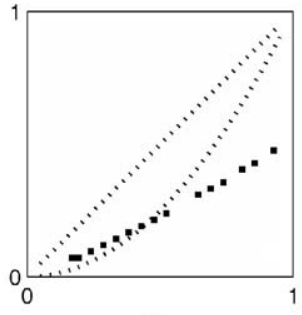

(C)

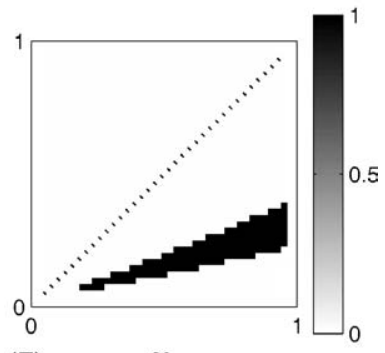

(F)

$\gamma_{1}$

Fig. 10. Summary of dynamics of competition between species with fruiting events, for the exploitative competition model (20)-(24) and the colonisation model (30)-(33). (A) Proportion of superior competitor after 1000 years in exploitative competition model. (B) Proportion of inferior competitor after 1000 years in exploitative competition model. (C) Coexistence or otherwise for exploitative competition model (black for coexistence, white for single species fixation). (D) Proportion of superior competitor after 1000 years in pre-emptive competition model. (E) Proportion of inferior competitor after 1000 years in pre-emptive competition model. (F) Coexistence or otherwise for exploitative coexistence model (black for coexistence, white for single species fixation). Parameter values: $m_{i}=0.0255, \beta_{1}=2, \beta_{2}=1$. The $\alpha_{i}$ are calculated using the expression for the average colonisation rates in Eq. (F.1), that is, $\alpha_{i}=\frac{1-\exp \left(-t_{\mathrm{f}} \beta_{i}\right)}{t_{\mathrm{f}} \beta_{i} c_{i}}$ with the $c_{i}$ being calculated from $c_{i}=\frac{m}{\gamma_{i}} \cdot t_{\mathrm{f}}$ is the average value of the length of time between fruiting events, here $t_{\mathrm{f}}=7$. Initial conditions for all simulations: $\left(p_{1}, p_{2}\right)=(0.475,0.475)$. The dotted lines in panel $\mathrm{C}$ indicate the boundaries of the parameter space for unstable coexistence in the model without fruiting events-see region 1 Fig. 1. The dotted lines in panel F correspond to the line of neutrally stable coexistence in the pre-emptive competition model-see region 1 in Fig. 2. The colourbar indicates the values represented by the shading in this figure.

Fig. 11, we present the results of 100 simulations of our exploitative competition (A) and colonisation (B) models for six species with the mean and standard deviation for each species after 100, 200, 500 and 1000 years. The model for six species is used for simulations in this section, and those following, as an example which captures all the dynamics of the model. It is clear that our colonisation model has much lower variability than our exploitative competition model, providing another reason to reassess the role of exploitative competition in tropical rain forests.

\subsubsection{Periodic fruiting events}

In order to assess the effect of the different components of our model, we conducted some more numerical experiments. Firstly, we considered the colonisation rates of the species. If we remove fruiting events from the model and compare constant and decaying colonisation rates for our competition and colonisation models, then we find that with only decaying colonisation rates, all the species die out. This is because the contribution from mortality overcomes the contribution from colonisation.

We have incorporated fruiting events into our models as an example of a disturbance event. The frequency of the events is periodic with a random time component determined by a random number generator in our simulations. We carried out further numerical experiments in order to determine whether temporal heterogeneity is a sufficient factor to reproduce long-term coexistence, or if randomness is required as well. When we considered strictly periodic disturbance events, we found that while the length of time to competitive exclusion is greater than in a model with no fruiting events, 

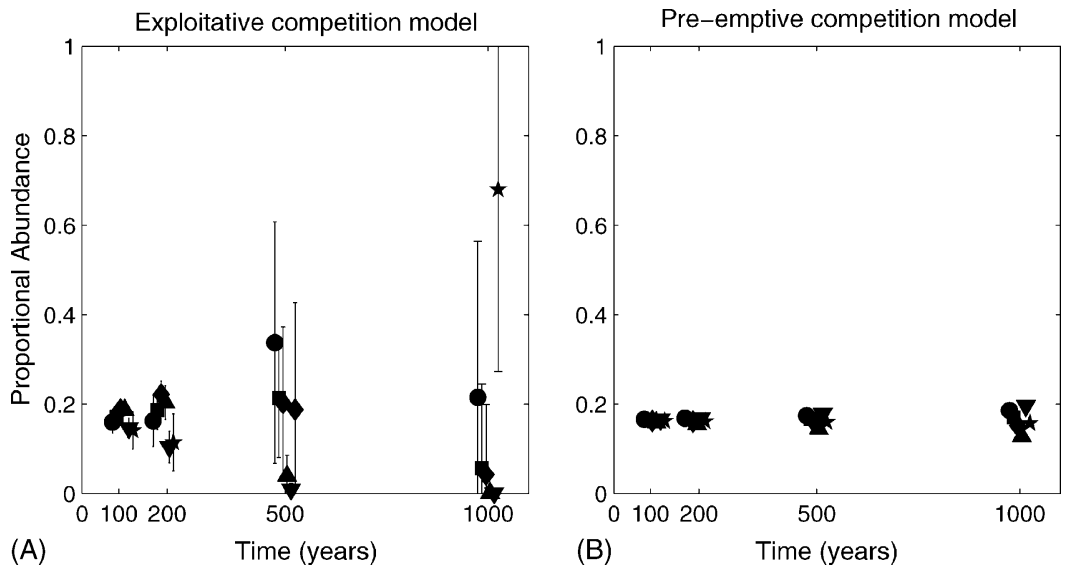

Fig. 11. Mean and standard deviation of proportional abundance for the six-species competition model (20)-(24) (A) and colonisation model (30)-(33) (B) for 100 simulations. The mean of each species is denoted by the following symbols: (1) ; (2) $\mathbf{\square}$; (3) $\mathbf{\text { ; }}$ (4) $\mathbf{\Delta}$; (5) $\mathbf{\nabla}$; (6) $\star$; and the standard deviation by the solid vertical lines. Note that the standard deviation for each species for the colonisation model in panel B is very small and although displayed, is obscured by the symbols denoting the mean values. The parameter values are given in Tables E.1 and E.2 $\left(m_{i}\right.$, $\alpha_{i}$ and $\beta_{i}$, Appendix E).

Exploitative competition, periodic fruiting events
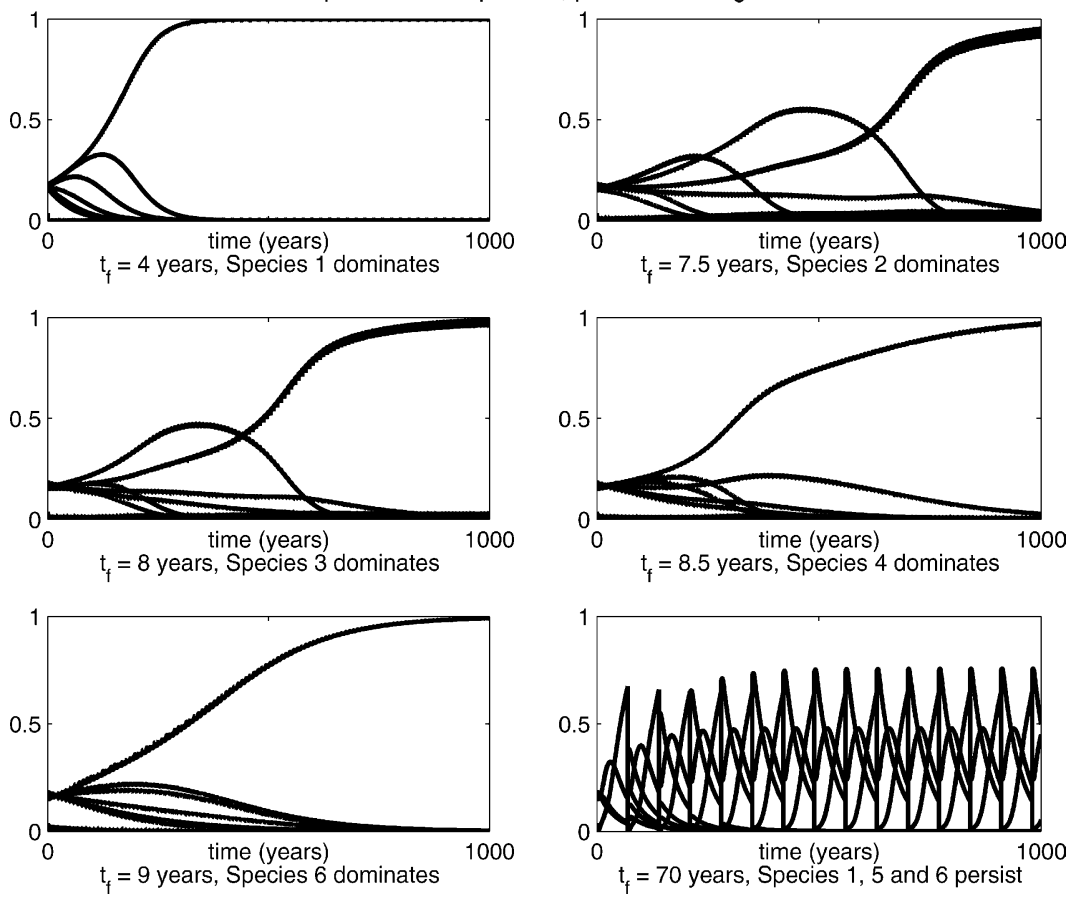

Fig. 12. Numerical experiments with periodic fruiting events for our competition model (20)-(24). The parameter values are given in Table E.1 $\left(m_{i}, \alpha_{i}\right.$ and $\beta_{i}$, Appendix E). 
competitive exclusion is not prevented (compare Fig. 4 with Fig. 12 and Fig. 7 with Fig. 13). Our simulations also demonstrate that if a fixed period between fruiting events is taken, one species always goes on to dominate the others (there is no variability between simulations). The particular species that dominates in each case depends on the period of the fruiting event interval. We would expect this to be the case as different species benefit from a particular period. We conclude that randomness in addition to temporal heterogeneity is required for long-term species coexistence. Some example results are shown in Fig. 12 (competition model) and Fig. 13 (colonisation model).

It is interesting to note that as the length of the interval between events increases, progressively lower ranked species in the competitive hierarchy dominate, until a threshold is reached $\left(t_{\mathrm{f}} \approx 10\right.$ for the colonisation model and $t_{\mathrm{f}} \approx 60$ for the competition model) and then species 1 dominates again. In each case once the interval between events is long enough, species 1 will dominate because the simulation proceeds as if there are no fruiting events. This is consistent with the results observed in Fig. 4 in the absence of fruiting events. Subsequent resetting of conditions does not alter the dominance of species 1 . Obviously, if the interval between fruiting events is greater than the average lifespan of an individual then even species 1 will become extinct.

\section{Discussion and conclusions}

Two new models for the dynamics of rain forest tree species have been proposed in this paper. The first incorporates a more realistic description of the process of competition between tropical rain forest tree seedlings than is embodied by Tilman's (1994) model. The deterministic model incorporates competition in the form of both exploitative and pre-emptive competition. In general, neither form of competition in the deterministic model leads to multiple species coexistence. One species ultimately excludes all others from colonising gaps.

However, linear stability analysis shows that there is bistability within certain parameter regimes. This
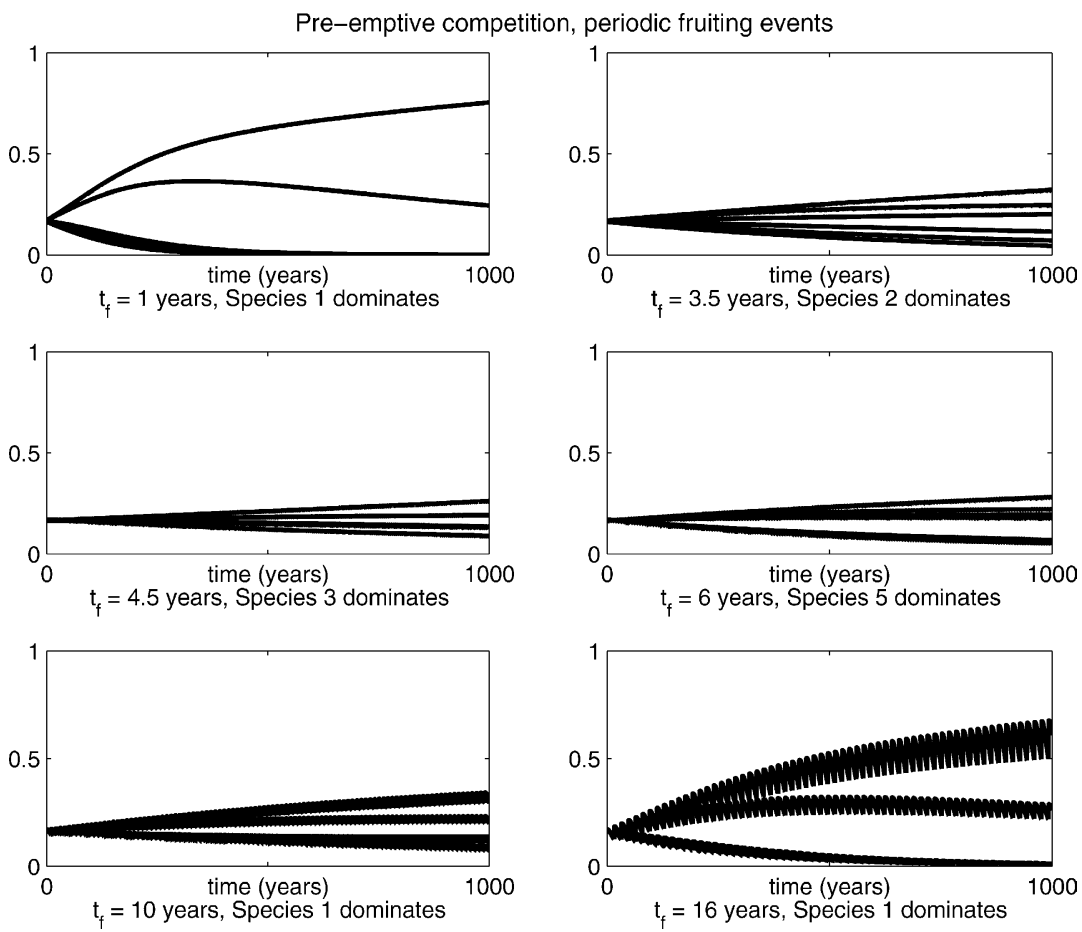

Fig. 13. Numerical experiments with periodic fruiting events for our colonisation model (30)-(33). The parameter values are given in Table E.2 $\left(m_{i}, \alpha_{i}\right.$ and $\beta_{i}$, Appendix E). 
means that there are two stable steady states with each species excluding the other in each one. The species that dominates the landscape is partially determined by the initial conditions of the system. If we imagine the spatial structure of the landscape as a patchwork, with different patches tending towards different species dominating in each patch, then we could envisage that immigration between patches (due to dispersal) might stop any species from going extinct in each patch. This mechanism has been invoked in Bell's neutral theory (2000) and in other patch models (Yu and Wilson, 2001). In practice this may lead to landscape level coexistence even if patch level coexistence is not maintained. In order to investigate such a possibility, dispersal rates would need to be measured and analysed. However, spatial segregation of species on the landscape has been criticised as to whether it can be defined as coexistence (Murrell and Law, 2003).

We conclude from the results for the two models described above that an additional factor needs to be incorporated in order to simulate the observed levels of species coexistence. We have included mastfruiting events, which are characteristic of Southeast Asian rain forests, occurring with a random frequency of between 3 and 11 years, triggered by the ENSO (Sakai et al., 1999; Yasuda et al., 1999). The seedling bank of each species decays over time between fruiting events so we changed the colonisation rates (constant until this point) to decay over time at a different rate for each species. The consequence of this is to allow each species to have a golden period during the interval between mast-fruiting events when it has the highest relative colonisation rate. This is a consequence of the strict trade-off between species producing many shortlived seedlings or few long-lived seedlings.

Kubo and Iwasa (1996) have previously proposed a model which allows for species coexistence if species have a different peak in their regeneration ability during the growing season. The phenological niche of the species also varies, those with non-overlapping niches are able to persist in their model framework, while those with broad overlapping niches do not. Their model is applicable in environments where annual synchronous recruitment takes place. However, in our system, this is not a valid assumption. The random supraannual intervals between subsequent fruiting events in our system allows for each species to encounter a favourable episode, subject to the condition that a sufficient number of adults survive the unfavourable conditions.

Mast-fruiting events have been introduced in both models (with exploitative and pre-emptive competition). Numerical simulation is used to generate the results, as mathematical analysis is difficult on such a complex system. Numerically, we observe that both models can exhibit coexisting species over some timescales. This effect is strongest in the stochastic model with pre-emptive competition (large numbers of species persist after $10^{5}$ years in Fig. 8). In order to achieve coexistence, in particular in the two-species case, we are forced to use unrealistic parameter values in the model with exploitative competition. Due to the ecological similarities between many climax rain forest tree species, on biological grounds we prefer the colonisation model. Additionally, a more thorough investigation of the parameter space (Fig. 10) leads us to the conclusion that coexistence is also possible over a wider range of circumstances (parameter sets) in the stochastic model with pre-emptive competition over the model with exploitative competition.

The random time component in the frequency of the mast-fruiting events is essential for stability. Periodic fruiting events were shown to result in single species dominance. The periodicity prevents some species from making use of their golden period. We believe that the random mast-fruiting events are an example of a time-varying nonlinearity that is often invoked as promoting species coexistence (Levins, 1979; Huston, 1979; Doyle, 1981). Other models, in response to Tilman's (1994) model invoke spatial structure in the form of variable density patches which enable species coexistence on the landscape scale (Yu and Wilson, 2001). We have demonstrated that a simpler alternative formulation, using endogenous temporal variability also allows coexistence.

Until now, many studies in the literature have either been empirically based and developed many ideas regarding community processes, or purely theoretical constructs (for example, the heteromyopia proposed by Murrell and Law (2003)). We used mathematical modelling as a tool to understand the maintenance of species diversity in the tropical rain forest. The aim of this study is not to develop more theory, but to incorporate knowledge gained empirically into the modelling framework. We were fortunate to have data on juvenile populations dynamics over 16 years to parameterise 
our models. There are already many simulation models designed to incorporate all possible factors and interactions, for example, the FORMIX3 model by Huth and Ditzer (2000) and TROLL model by Chave (1999). Conversely, the models proposed here are unique such that they express the most parsimonious representation of the relevant biological phenomena. The modelling framework allowed us to add or remove components and allowed us to disentangle the effects of specific features of the model.

The models presented in this paper incorporate several simplifying assumptions. This is an important general criticism made by experimentalists of modellers (Peters, 1991). It is important to assess whether relaxing some of the assumptions will yield more realistic results in terms of species coexistence and the maintenance of diversity in tropical rain forests. The model framework used so far has only taken into account adult trees. The colonisation rates used in our models assume an instantaneous change from juvenile to adult, while the success of an individual in a gap is likely to be a function of the presence of juveniles of other species, rather than the adults. The dynamics of seedling and sapling populations are no doubt important (Whitmore and Brown, 1996) in the forest structure and a stagestructured model would investigate these. In future, we hope to examine the impact on species coexistence via the mechanism of juvenile dynamics and adult dynamics operating over different temporal scales. The model framework we have developed allows for additional decoration to be added as needed.

We believe that the results in this paper have general implications for all tropical rain forests in that it suggests that the type of trade-off proposed by Tilman (1994) is not a sufficient condition for species coexistence in an ecosystem where competitive displacement of one species by another is unlikely. It also suggests that time-varying nonlinearities are important in such systems.

\section{Acknowledgements}

The authors are pleased to acknowledge the financial support of the following organisations: a studentship from EPSRC and from NERC for CJB; a Career Development Fellowship from the Medical Research Council for DJG; which has allowed them to undertake this research. We acknowledge the following for permission to conduct research in the Danum Valley, Sabah: Yayasan Sabah (Forestry Division), The Danum Valley Management Committee, The State Secretary (Internal Affairs \& Research Office), Sabah Chief Minister's Department, and the Economic Planning Unit of the Prime Minister's Department, Kuala Lumpur. We thank Mr. Bahaman Ahmad (Tropical Biology and Conservation Unit, University of Malaysia Sabah), Mr. Tom Clements for invaluable assistance in the field, Dr. Jonathan Whiteley and Dr. David Sumpter for helpful discussions, Prof. Michael Huston for very constructive and enlightening comments. This paper is based on material collected whilst CJB was a participant in The Royal Society's SE Asia Rainforest Research Programme, project number 184 .

\section{Appendix A. Eigenvalues for coexistence steady state for model Eqs. (7) and (8)}

The eigenvalues of the Jacobian matrix $\boldsymbol{J}_{2}$ in (9) can be calculated using Maple (Waterloo Maple Software, 2001) to yield:

$$
\begin{aligned}
\hat{\lambda}_{1}= & \frac{1}{2 \gamma_{1}^{2}}\left(-c_{1} \gamma_{1}^{2}+c_{2} \gamma_{2} \gamma_{1}^{2}+c_{1} \gamma_{2} \gamma_{1}-c_{2} \gamma_{2}^{2}+\sqrt{\left(c_{1}^{2} \gamma_{1}^{4}+2 c_{1} \gamma_{1}^{4} c_{2} \gamma_{2}-2 c_{1}^{2} \gamma_{1}^{3} \gamma_{2}+2 c_{1} \gamma_{1}^{2} c_{2} \gamma_{2}^{2}+c_{2}^{2} \gamma_{2}^{2} \gamma_{1}^{4}\right.}\right. \\
& \left.\times \sqrt{\left.-2 c_{2} \gamma_{2}^{2} \gamma_{1}^{3} c_{1}-2 c_{2}^{2} \gamma_{2}^{3} \gamma_{1}^{2}+c_{1}^{2} \gamma_{2}^{2} \gamma_{1}^{2}-2 c_{1} \gamma_{2}^{3} \gamma_{1} c_{2}+c_{2}^{2} \gamma_{2}^{4}-4 \gamma_{1}^{6} c_{1} c_{2}+4 \gamma_{1}^{5} c_{1} c_{2} \gamma_{2}\right)}\right), \\
\hat{\lambda}_{2}= & \frac{1}{2 \gamma_{1}^{2}}\left(-c_{1} \gamma_{1}^{2}+c_{2} \gamma_{2} \gamma_{1}^{2}+c_{1} \gamma_{2} \gamma_{1}-c_{2} \gamma_{2}^{2}-\sqrt{\left(c_{1}^{2} \gamma_{1}^{4}+2 c_{1} \gamma_{1}^{4} c_{2} \gamma_{2}-2 c_{1}^{2} \gamma_{1}^{3} \gamma_{2}+2 c_{1} \gamma_{1}^{2} c_{2} \gamma_{2}^{2}+c_{2}^{2} \gamma_{2}^{2} \gamma_{1}^{4}\right.}\right. \\
& \left.\times \sqrt{\left.-2 c_{2} \gamma_{2}^{2} \gamma_{1}^{3} c_{1}-2 c_{2}^{2} \gamma_{2}^{3} \gamma_{1}^{2}+c_{1}^{2} \gamma_{2}^{2} \gamma_{1}^{2}-2 c_{1} \gamma_{2}^{3} \gamma_{1} c_{2}+c_{2}^{2} \gamma_{2}^{4}-4 \gamma_{1}^{6} c_{1} c_{2}+4 \gamma_{1}^{5} c_{1} c_{2} \gamma_{2}\right)}\right)
\end{aligned}
$$




\section{Appendix B. Finding the stability of the coexistence steady state for the $\boldsymbol{n}$-species competition model}

Initially, we need to calculate the Jacobian for the multiple species system. From Eq. (13), we find that:

$$
\begin{aligned}
\frac{\partial}{\partial p_{i}}\left(\frac{\mathrm{d} p_{i}}{\mathrm{~d} t}\right)= & c_{i}\left(1-\sum_{k=1}^{n} p_{k}\right)\left(1-\sum_{k=1}^{i-1} p_{k}\right) \\
& -c_{i} p_{i}\left(1-\sum_{k=1}^{i-1} p_{k}\right)-c_{i} \gamma_{i},
\end{aligned}
$$$$
\frac{\partial}{\partial p_{j}}\left(\frac{\mathrm{d} p_{i}}{\mathrm{~d} t}\right)=-c_{i} p_{i}\left(2-\sum_{k=1}^{i-1} p_{k}-\sum_{k=1}^{n} p_{k}\right),
$$$$
\text { for } j<i \text {, }
$$

$$
\frac{\partial}{\partial p_{j}}\left(\frac{\mathrm{d} p_{i}}{\mathrm{~d} t}\right)=-c_{i} p_{i}\left(1-\sum_{k=1}^{i-1} p_{k}\right), \quad \text { for } \quad j>i
$$

Considering first the case $i=1$, setting $\frac{\mathrm{d} p_{1}}{\mathrm{~d} t}=0$ in (13), we obtain either $p_{1}=0$ or $1-\sum_{i=1}^{n} p_{i}=\gamma_{1}$. Substituting this into Eq. (13) for $i \geq 2$, we see that $1-\sum_{k=1}^{i-1} p_{k}=\frac{\gamma_{i}}{\gamma_{1}}$, and substituting this in turn into Eqs. (B.1)-(B.3), the entries of the Jacobian become:

$$
\frac{\partial}{\partial p_{j}}\left(\frac{\mathrm{d} p_{i}}{\mathrm{~d} t}\right)=-c_{i} p_{i}\left(\frac{\gamma_{i}}{\gamma_{1}}+\gamma_{1}\right), \quad \text { for } \quad j<i,
$$

$$
\frac{\partial}{\partial p_{j}}\left(\frac{\mathrm{d} p_{i}}{\mathrm{~d} t}\right)=-c_{i} p_{i} \frac{\gamma_{i}}{\gamma_{1}}, \quad \text { for } \quad j \geq i
$$

From (B.4) and (B.5), we find that the general Jacobian matrix, $\boldsymbol{J}_{n}$, takes the form:

$$
\boldsymbol{J}_{n}=\left(\begin{array}{ccccc}
a_{1} & a_{1} & a_{1} & \cdots & a_{1} \\
\alpha_{2} & a_{2} & a_{2} & \cdots & a_{2} \\
\alpha_{3} & \alpha_{3} & a_{3} & \cdots & a_{3} \\
\vdots & \vdots & & \ddots & \vdots \\
\alpha_{n} & \alpha_{n} & \cdots & & a_{n}
\end{array}\right) .
$$

To derive results on the stability properties of the steady states we use the following result and corollary.

Result 1. There exists at least one eigenvalue of the stability matrix, $\boldsymbol{J}_{n}$, in the right half of the complex plane.

Corollary 2. The system of Eq. (13) for $n>1$ has at most one possible stable steady state. This is the steady state in which only one of the species is non-zero and all the other species die out.

Proof 1 (Proof of Result 1). The $\boldsymbol{L} \boldsymbol{U}$ decomposition of $\boldsymbol{J}_{n}$ is:

$$
\begin{aligned}
\left|\boldsymbol{J}_{n}\right|=\boldsymbol{L} \boldsymbol{U} & =\left(\begin{array}{ccccc}
1 & 0 & 0 & \cdots & 0 \\
\frac{\alpha_{2}}{a_{1}} & 1 & 0 & \cdots & 0 \\
\frac{\alpha_{3}}{a_{1}} & 0 & 1 & \cdots & 0 \\
\vdots & \vdots & \vdots & \ddots & \cdots \\
\frac{\alpha_{n}}{a_{1}} & 0 & 0 & \cdots & 1
\end{array}\right) \\
& \times\left(\begin{array}{cccccc}
a_{1} & a_{1} & & \cdots & \cdots & a_{1} \\
0 & a_{2}-\alpha_{2} & a_{2} & \cdots & a_{2} \\
0 & 0 & a_{3}-\alpha_{3} & \cdots & a_{3} \\
\vdots & \vdots & & & \ddots & \vdots \\
0 & 0 & 0 & \cdots & a_{n}-\alpha_{n}
\end{array}\right) .
\end{aligned}
$$


(The $\boldsymbol{L} \boldsymbol{U}$ decomposition of a matrix is its equivalent expression as a product of a lower triangular matrix, a matrix with zeros above the diagonal, $\boldsymbol{L}$, and an upper triangular matrix, a matrix with zeros below the diagonal, $\boldsymbol{U}$.) From the $\boldsymbol{L} \boldsymbol{U}$ decomposition of $\boldsymbol{J}_{n}$ it is clear that:

$\left|\boldsymbol{J}_{n}\right|=a_{1}\left(a_{2}-\alpha_{2}\right)\left(a_{3}-\alpha_{3}\right) \cdots\left(a_{n}-\alpha_{n}\right)<0$.

Since $p_{i} \geq 0$ and $0<\gamma_{1}^{2}<\gamma_{n}<\cdots<\gamma_{2}<\gamma_{1}<1$, we have that $a_{1}<0$, and $a_{i}-\alpha_{i} \geq 0$ for all $i$. We know that $\boldsymbol{J}_{n}=(-1)^{n} \prod_{i=1}^{n} \lambda_{i}$. Combining this with (B.8), we see that there is always at least one eigenvalue with $\operatorname{Re}\left(\lambda_{i}\right)>0$ for all values of $n$.

Proof 2 (Proof of Corollary 2). From Result 1, we know that the steady state with all $n$ species having a non-zero abundance is unstable. Hence, the system reduces to that of $(n-1)$ species. Similarly this reduces by one dimension, until we are left with a twospecies system. In Section 3.1, we have shown that the only stable steady state is for one species dominating and the other decaying to zero. In the onespecies case, the only possible stable steady state is for $p_{1}=1-\frac{m_{1}}{c_{1}}>0$. The steady state $\left(p_{1}, 0, \ldots, 0\right)$ for $n$ species is stable since all its eigenvalues (which can be easily shown to be $\lambda_{1}=2 c_{1} \gamma_{1}\left(\gamma_{1}-1\right), \lambda_{i}=$ $\left.c_{i}\left(\gamma_{1}^{2}-\gamma_{i}\right), i=2, \ldots, n\right)$ are less than zero since $0<$ $\gamma_{1}^{2}<\gamma_{i}<\gamma_{1}<1$ for all $i$.

\section{Appendix C. Calculating the timescale for decay}

If we assume that a population's decay is exponential, then we can calculate the time for the population to be reduced to half its initial value: its half-life. In the same way, we can calculate the time taken for the population to reduce to $1 \%$ of its initial value:

$t_{1 \%}=\frac{\ln \left(100 / y_{0}\right)}{\lambda}$,

where $y_{0}$ is the initial value of the population and $\lambda$ is the associated eigenvalue. In our models, we have many species interacting and their timescale for dominance is taken to be:

$t_{1 \%}=\frac{\ln \left(100 / y_{0}\right)}{\min _{i \in[1, n]}\left|\lambda_{i}\right|}$,

and the timescale for $50 \%$ of species to decay is:

$t_{1 \%}^{50 \%}=\frac{\ln \left(100 / y_{0}\right)}{\operatorname{median}_{i \in[1, n]}\left|\lambda_{i}\right|}$.

The eigenvalues, $\lambda_{i}$ used in these calculations are those for the trivial steady state:

$\left(0, \ldots, 0, p_{j}, 0, \ldots, 0\right), \quad$ for $\quad j \in[1, n]$.

\section{Appendix D. Balance of proliferation and death of species}

We decided to determine, as a guide, the ratio of $\alpha_{i}: \beta_{i}$ demanding that the proportions of each species should return to their initial proportions after an average fruiting interval, $t_{\mathrm{f}}$. In this way, we are searching for a balance of proliferation and death of each species. Assuming that $p_{i}$ and $g$ are constant (as there are only small changes in proportions), we then find for the twospecies model:

$\int_{0}^{t_{\mathrm{f}}} p_{1} \mathrm{~d} t=p_{1} g \alpha_{1} \int_{0}^{t_{\mathrm{f}}} \mathrm{e}^{-\beta_{1} t} \mathrm{~d} t-p_{1} m_{1} \int_{0}^{t_{\mathrm{f}}} \mathrm{d} t$.

For $p_{1} \neq 0$, we obtain:

$\frac{\alpha_{1}}{\beta_{1}}=\frac{\left(1+m_{1}\right) t_{\mathrm{f}}}{g}$,

and similarly for species 2 :

$\frac{\alpha_{2}}{\beta_{2}}=\frac{\left(1+m_{2}\right) t_{\mathrm{f}}}{g\left(1-p_{1}\right)}$.

For the $i$ th species, the ratio of $\alpha_{i}$ to $\beta_{i}$ is given by:

$\frac{\alpha_{i}}{\beta_{i}}=\frac{\left(1+m_{i}\right) t_{\mathrm{f}}}{g\left(1-\sum_{j=1}^{i-1} p_{j}\right)}$.

These expressions are used as a starting point to find the correct order of magnitude of the parameter values. For example, for two species, we can see the left-hand side of (D.2) equals 312 (from the parameter values used for the two-species exploitative competition model, Table E.1) and the right-hand side equals 239; and for (D.3), the left-hand side equals 180 and the right-hand side equals 455 . 
Appendix E.

Parameter values for numerical simulations

Table E.1

Parameter values and initial conditions for the numerical simulations shown in Figs. 4 and 5

\begin{tabular}{|c|c|c|c|c|c|c|}
\hline Panel & Species & $\alpha_{i}\left(\right.$ seedlings tree ${ }^{-1}$ year $\left.^{-1}\right)$ & $\beta_{i}\left(\right.$ year $\left.^{-1}\right)$ & $\overline{c_{i}}\left(\right.$ seedlings tree $^{-1}$ year $\left.^{-1}\right)$ & $m_{i}\left(\right.$ year $\left.^{-1}\right)$ & $\begin{array}{l}\text { Initial } \\
\text { conditions }\end{array}$ \\
\hline \multirow[t]{2}{*}{ Top } & $p_{1}$ & 500 & 1.6 & 44.6422 & 0.0255 & 0.475 \\
\hline & $p_{2}$ & 9 & 0.05 & 7.5937 & 0.0255 & 0.475 \\
\hline \multirow[t]{6}{*}{ Middle } & $p_{1}$ & 385 & 1.3930 & 39.4808 & 0.0255 & 0.1603 \\
\hline & $p_{2}$ & 325 & 1.1113 & 41.7611 & 0.0255 & 0.1603 \\
\hline & $p_{3}$ & 275 & 0.89 & 44.0543 & 0.0255 & 0.1603 \\
\hline & $p_{4}$ & 150 & 0.5785 & 36.3959 & 0.0255 & 0.1603 \\
\hline & $p_{5}$ & 90 & 0.3749 & 31.8088 & 0.0255 & 0.1603 \\
\hline & $p_{6}$ & 60 & 0.179 & 34.2069 & 0.0255 & 0.1603 \\
\hline \multirow[t]{2}{*}{ Bottom } & $p_{1}$ & 145 & 1.2 & 17.2580 & 0.0255 & 0.0053 \\
\hline & $p_{i}(i=2,180)$ & $145-\frac{75}{179}(i-1)$ & $1.2-\frac{0.4}{179}(i-1)$ & $\frac{\alpha_{i}}{\beta_{i} t_{\mathrm{f}}}\left(1-\mathrm{e}^{-\beta_{i} t_{\mathrm{f}}}\right)$ & 0.0255 & 0.0053 \\
\hline
\end{tabular}

Table E.2

Parameter values and initial conditions for the numerical simulations shown in Figs. 7 and 8

\begin{tabular}{|c|c|c|c|c|c|c|}
\hline Panel & Species & $\alpha_{i}\left(\right.$ seedlings tree ${ }^{-1}$ year $\left.^{-1}\right)$ & $\beta_{i}\left(\right.$ year $\left.^{-1}\right)$ & $\bar{c}_{i}\left(\right.$ seedlings tree ${ }^{-1}$ year $\left.^{-1}\right)$ & $m_{i}\left(\right.$ year $\left.^{-1}\right)$ & $\begin{array}{l}\text { Initial } \\
\text { conditions }\end{array}$ \\
\hline \multirow[t]{2}{*}{ Top } & $p_{1}$ & 130 & 1.2 & 15.4727 & 0.0255 & 0.475 \\
\hline & $p_{2}$ & 25 & 0.5 & 6.9272 & 0.0255 & 0.475 \\
\hline \multirow[t]{6}{*}{ Middle } & $p_{1}$ & 145 & 1.2239 & 16.9216 & 0.0255 & 0.1617 \\
\hline & $p_{2}$ & 135 & 1.1647 & 16.5538 & 0.0255 & 0.1617 \\
\hline & $p_{3}$ & 90 & 0.9144 & 14.0374 & 0.0255 & 0.1617 \\
\hline & $p_{4}$ & 83 & 0.8796 & 13.4516 & 0.0255 & 0.1617 \\
\hline & $p_{5}$ & 73 & 0.8179 & 12.7088 & 0.0255 & 0.1617 \\
\hline & $p_{4}$ & 70 & 0.8047 & 12.3825 & 0.0255 & 0.1617 \\
\hline \multirow[t]{2}{*}{ Bottom } & $p_{1}$ & 145 & 1.2 & 17.2580 & 0.0255 & 0.0053 \\
\hline & $p_{i}(i=2,180)$ & $145-\frac{75}{179}(i-1)$ & $1.2-\frac{0.4}{179}(i-1)$ & $\frac{\alpha_{i}}{\beta_{i} t_{\mathrm{f}}}\left(1-\mathrm{e}^{-\beta_{i} t_{\mathrm{f}}}\right)$ & 0.0255 & 0.0053 \\
\hline
\end{tabular}

\section{Appendix F. Determination of average values of the colonisation rates}

In order to compare the models without and with fruiting events included, we introduce the definition of $\bar{c}_{i}$, that is the average of $c_{i}$ over the fruiting interval, i.e.

$\bar{c}_{i}=\frac{1}{t_{\mathrm{f}}} \int_{0}^{t_{\mathrm{f}}} \alpha_{i} \mathrm{e}^{-\beta_{i} t} \mathrm{~d} t$.

\section{References}

Bampfylde, C.J., 1999. Modelling rainforests. M.Sc. thesis, Mathematical Institute, University of Oxford, Oxford.

Bazzaz, F.A., Pickett, S.T.A., 1980. Physiological ecology of tropical succession: a comparative review. Annu. Rev. Ecol. Syst. 11, 287-310.

Bell, G., 2000. The distribution of abundance in neutral communities. Am. Nat. 155 (5), 606-617.

Brown, N.D., Press, M., Bebber, D., 1999. Growth and survivorship of dipterocarp seedlings: differences in shade persistence create 
a special case of dispersal limitation. Philos. Trans. R. Soc. Lond. B 354, 1847-1855.

Burgess, P.F., 1972. Studies on the regeneration of the hill forest of the Malay Peninsular. The phenology of dipterocarps. Malays. For. 35, 103-123.

Byrne, G.D., Hindmarsh, A.C., 1987. Stiff ODE solvers: a review of current and coming attractions. J. Comput. Phys. 70 (1), 1-62.

Callard, R.E., Stark, J., Yates, A.J., 2003. Fratricide: a mechanism for T memory-cell homeostasis. Trends Immunol. 24 (7), 370375.

Chave, J., 1999. Study of structural, successional and spatial patterns in tropical rain forests using TROLL, a spatially explicit forest model. Ecol. Model. 124 (2-3), 233-254.

Chesson, P.L., Warner, R.R., 1981. Environmental variability promotes coexistence in lottery competitive-systems. Am. Nat. 117, 923-943.

Clark, J.S., Beckage, B., Camill, P., Cleveland, B., Hillerislambers, J., Lichter, J., McLachlan, J., Mohan, J., Wyckoff, P., 1999. Interpreting recruitment limitation in forests. Am. J. Bot. 86, 1-16.

Connell, J.H., 1978. Diversity in tropical rain forests and coral reefs. Science 199, 1302-1310.

Curran, L.M., Caniago, I., Paoli, G.D., Astianti, D., Kusneti, M., Leighton, M., Nirarita, C.E., Haeruman, H., 1999. Impact of El Niño and logging on canopy tree recruitment in Borneo. Science 286 (5447), 2184-2188.

Delissio, L.J., Primack, R.B., Hall, P., Lee, H.S., 2002. A decade of canopy-tree seedling survival and growth in two Bornean rain forests: persistence and recovery from suppression. J. Trop. Ecol. 18 (5), 645-658.

Doyle, T.W., 1981. The role of disturbance in the gap dynamics of a montane rain forest: an application of a tropical forest succession model. In: Botkin, D.B., Shugart, H.H., West, D.C. (Eds.), Forest Succession: Concepts and Application. Springer-Verlag, New York, pp. 56-73.

Fox, J.E.D., 1976. Constraints on the natural regeneration of tropical moist forest. For. Ecol. Manage. 1, 37-65.

Freckleton, R., Watkinson, A., 2000. On detecting and measuring competition in spatially structured plant communities. Ecol. Lett. 3 (5), 423-432.

Freckleton, R., Watkinson, A., 2001. Predicting competition coefficients for plant mixtures: reciprocity, transitivity and correlations with life-history traits. Ecol. Lett. 4 (4), 348-357.

Hartshorn, G.S., 1978. Tree falls and tropical forest dynamics. In: Tomlinson, P.B., Zimmermann, Z.H. (Eds.), Tropical Trees as Living Systems. Cambridge University Press, Cambridge, pp. 617-638.

Howlett, B.E., Davidson, D.W., Appanah, S., Khoo, K.C., 1996. Dipterocarp seed and seedling performance in secondary logged forests dominated by Macaranga spp. In: Proceedings of the Fifth Round Table Conference on Dipterocarps, Forest Research Institute Malaysia (FRIM), Kuala Lumpur, Malaysia, pp. 256266.

Hubbell, S.P., 1998. The maintenance of diversity in a neotropical tree community: conceptual issues, current evidence, and challenges ahead. In: Dallmeier, F., Comiskey, J.A. (Eds.), Forest Biodiversity Research, Monitoring and Modeling. UNESCO/Parthenon Publishing Group, Paris/Carnforth, Lancashire, pp. 17-44.
Hubbell, S.P., 2001. The Unified Neutral Theory of Biodiversity and Biogeography. Princeton University Press, Princeton, NJ/Oxford.

Hubbell, S.P., Foster, R.B., 1986. Community ecology. In: Biology, Chance and History, and the Structure of Tropical Tree Communities. Harper Row, New York, pp. 314-324.

Hubbell, S.P., Foster, R.B., O’Brien, S.T., Harms, K.E., Condit, R., Wechsler, B., Wright, S.J., Loo de Lao, S., 1999. Light-gap disturbances, recruitment limitation, and tree diversity in a neotropical forest. Science 283, 554-557.

Hurtt, G.C., Pacala, S.W., 1995. The consequences of recruitment limitation: reconciling chance, history and competitive differences between plants. J. Theor. Biol. 176, 1-12.

Huston, M.A., 1979. A general hypothesis of species diversity. Am. Nat. 113, 81-101.

Hutchinson, G.E., 1961. The paradox of the plankton. Am. Nat. 95, 137-145.

Huth, A., Ditzer, T., 2000. Simulation of the growth of a lowland dipterocarp rain forest with FORMIX3. Ecol. Model. 134 (1), $1-25$.

Jordan, D.W., Smith, P., 1988. Nonlinear Ordinary Differential Equations, second ed. Clarendon Press, Oxford.

Keddy, P.A., Shipley, B., 1989. Competitive hierarchies in herbaceous plant communities. Oikos 54, 234-241.

Kochummen, K.M., 1996. Natural plant succession after farming in Sg. Kroh. Malays. For. 29, 170-181.

Kubo, T., Iwasa, Y., 1996. Phenological pattern of tree regeneration in a model for forest species diversity. Theor. Popul. Biol. 49, 90-117.

Lambert, F.R., Marshall, A.G., 1991. Keystone characteristics of bird-dispersed Ficus in a Malaysian lowland rain forest. J. Ecol. 79, 793-809.

Levins, R., 1969. Some demographic and genetic consequences of environmental heterogeneity for biological control. Bull. Entomol. Sci. Am. 15, 237-240.

Levins, R., 1979. Coexistence in a variable environment. Am. Nat. 114, 765-783.

Levins, R., Culver, D., 1971. Regional coexistence of species and competition between rare species. Proc. Natl. Acad. Sci. U.S.A. 68, 1246-1248.

MathWorks Inc., 2002. MATLAB: the language of technical computing, version 6.5. MathWorks Inc., Natick, MA.

McPhaden, M.J., 1999. Climate oscillations: genesis and evolution of the 1997-1998 El Niño. Science 283 (5404), 950-957.

McPhaden, M.J., 1999. El Niño: the child prodigy of 1997-1998. Nature 398 (6728), 559-561.

Murrell, D.J., Law, R., 2003. Heteromyopia and the spatial coexistence of similar competitors. Ecol. Lett. 6 (1), 48-59.

Newstrom, L.E., Frankie, G.W., Baker, H.G., 1994. A new classification for plant phenology based on flowering patterns in lowland tropical rain-forest trees at La-Selva, Costa-Rica. Biotropica 26, 141-159.

Peters, R.H., 1991. A Critique for Ecology. Cambridge University Press, Cambridge.

Press, M.C., Brown, N.D., Barker, M.G., Zipperlin, S.W., 1996. Photosynthetic responses to light in tropical forest tree seedlings. In: Swaine, M.D., UNESCO (Eds.), The Ecology of Tropical 
Forest Tree Seedlings. UNESCO/Parthenon Publishing Group, Paris/Carnforth, Lancashire, pp. 41-58.

Rees, M., Bergelson, J., 1997. Asymmetric light competition and founder control in plant communities. J. Theor. Biol. 184, 353358.

Roughgarden, J., Iwasa, Y., 1986. Dynamics of a metapopulation with space-limited subpopulations. Theor. Popul. Biol. 29, 235261.

Sakai, S., Momose, K., Yumoto, T., Nagamitsu, T., Nagamasu, H., Hamid, A.A., Nakashizuka, T., 1999. Plant reproductive phenology over four years including an episode of general flowering in a lowland dipterocarp forest, Sarawak, Malaysia. Am. J. Bot. 86 (10), 1414-1436.

Shampine, L.F., Reichelt, M.W., 1997. The MATLAB ODE suite. SIAM J. Sci. Comput. 18 (1), 1-22.

Still, M.J., 1996. Rates of growth and mortality in three groups of dipterocarp seedlings in Sabah, Malaysia. In: Swaine, M.D. (Ed.), The Ecology of Tropical Forest Tree Seedlings. UNESCO/Parthenon Publishing Group, Paris/Carnforth, Lancashire, pp. 315-330.

Svenning, J.C., 1999. Microhabitat specialization in a species-rich palm community in Amazonian Ecuador. J. Ecol. 87, 55-65.

Swaine, M.D., Whitmore, T.C., 1988. On the definition of ecological species groups in tropical rain forests. Vegetatio 75, 81-86.

Tilman, D., 1994. Competition and biodiversity in spatially structured habitats. Ecology 75, 2-16.
Trefethen, L.N., 1996. Finite difference and spectral methods for ordinary and partial differential equations. Online http://www.web.comlab.ox.ac.uk/oucl/work/nick.trefethen/ pdetext.html.

Waterloo Maple Software, 2001. Maple 7. Brooks/Cole, Pacific Grove, California.

Webster, P.J., Palmer, T.N., 1997. The past and the future of El Niño. Nature 390 (6660), 562-564.

Weigelt, A., Jolliffe, P., 2003. Indices of plant competition. J. Ecol. 91 (5), 707-720.

Whitmore, T.C., 1984. Tropical Rain Forests of the Far East, second ed. Clarendon Press, Oxford.

Whitmore, T.C., 1998. An Introduction to Tropical Rain Forests, second ed. Oxford University Press, Oxford.

Whitmore, T.C., Brown, N.D., 1996. Dipterocarp seedling growth in rain forest canopy gaps during six and a half years. Philos. Trans. R. Soc. Lond. B 351, 1195-1203.

Yasuda, M., Matsumoto, J., Osada, N., Ichikawa, S., Furukawa, A., Nik, A.R., Manokaran, N., 1999. The mechanism of general flowering in Dipterocarpaceae in the Malay Peninsula. J. Trop. Ecol. 15, 437-449.

Yates, A., Callard, R., 2001. Cell death and the maintenance of immunological memory. Discrete Cont. Dyn. B 1 (1), 43-60.

Yu, D.W., Wilson, H.B., 2001. The competition-colonization tradeoff is dead: long live the competition-colonization trade-off. Am. Nat. 158 (1), 49-63. 